\title{
ArcheoSciences
}

Revue d'archéométrie

\section{La cité médiévale d'Aigues-Mortes (Gard) : nouvelles données géomorphologiques et archéologiques sur un site méconnu}

The medieval fortress of Aigues-Mortes: geomorphologic and archaeologic new data at an unknown site

Tony Rey, Christian Markiewicz, Patrick Florençon et Anne-Sophie Lartigot-Campin

\section{OpenEdition}

Journals

Édition électronique

URL : https://journals.openedition.org/archeosciences/4504

DOI : $10.4000 /$ archeosciences. 4504

ISBN : 978-2-7535-4778-0

ISSN : 2104-3728

Éditeur

Presses universitaires de Rennes

\section{Édition imprimée}

Date de publication : 31 décembre 2015

Pagination : 201-215

ISBN : 978-2-7535-4776-6

ISSN : 1960-1360

Référence électronique

Tony Rey, Christian Markiewicz, Patrick Florençon et Anne-Sophie Lartigot-Campin, « La cité médiévale d'Aigues-Mortes (Gard) : nouvelles données géomorphologiques et archéologiques sur un site méconnu », ArcheoSciences [En ligne], 39 | 2015, mis en ligne le 31 décembre 2017, consulté le 01 février 2022. URL : http://journals.openedition.org/archeosciences/4504; DOI : https://doi.org/ 10.4000/archeosciences.4504 


\title{
La cité médiévale d'Aigues-Mortes (Gard) : nouvelles données géomorphologiques et archéologiques sur un site méconnu
}

\author{
The Medieval Fortress of Aigues-Mortes: \\ Geomorphologic and Archaeologic New Data at an Unknown Site
}

\author{
Tony Rey ${ }^{a}$, Christian Markiewicz ${ }^{b}$, Patrick FlorençON ${ }^{c}$ \\ et Anne-Sophie Lartigot-Campin ${ }^{\mathrm{d}}$
}

\begin{abstract}
Résumé : Depuis 2013, la cité d'Aigues-Mortes fait l'objet de recherches dans le cadre d'un Projet Collectif de Recherche "géoarchéologie des ports médiévaux d'Aigues-Mortes ". Le PCR regroupe archéologues, historiens, palynologues, géomorphologues qui s'intéressent à la fondation de cette cité portuaire. Les études portent sur la dynamique paysagère au Moyen Âge, le fonctionnement du port, les archives, l'inventaire des vestiges, les échanges commerciaux, les techniques de construction... Aigues-Mortes était au XIII ${ }^{\mathrm{e}}$ siècle une cité portuaire au sein d'un paysage aux ambiances lagunaires, marines et palustres. Le port d'Aigues-Mortes s'est colmaté rapidement. Moins de 50 ans après sa fondation, des dragages ont été réalisés mais en vain. Les pressions humaines et climatiques (Petit âge de glace) peuvent expliquer cette hyper-sédimentation. Le rivage lagunaire se situait à une vingtaine de mètres des fortifications. Depuis la rive, on accédait à la cité sur un sol constitué par plusieurs remblais afin de surélever un terrain trop humide. À la base du rempart, les constructions présentent une élévation en moellons à bossage qui prend appui sur une structure saillante. L'ensemble forme un radier de pose. Les données archéologiques révèlent aussi la richesse de la production de céramiques et de faïences de l'Uzège et des échanges commerciaux avec la Catalogne.
\end{abstract}

Abstract: Since 2013, the city of Aigues-Mortes is being investigated as part of a Collective Research Project "geoarchaeology medieval harbour of AiguesMortes". The PCR brings together archaeologists, historians, palynologists, geomorphologists interesting in the foundation of this harbour city. The studies focus on landscape dynamics during the Middle Age, the operation of the harbour, archives, inventory of remains, construction techniques... In the $13^{\text {th }}$ century Aigues-Mortes was a harbour city in within a landscape of brackish waters, marine and wetland. The harbour of Aigues-Mortes is quickly filled. Less than 50 years after its founding, dredging has been made in vain. The human and climatic pressures (Little Ice Age) may explain this hypersedimentation. The lagoon shore was at about twenty meters fortifications. From the shore, one reached the city on a floor consisting of several backfills to raise too wet ground. Under the wall, the base have an elevation in rubble boss which builds on a protruding structure. The assembly forms a upron. Archaeological data also show the importance of the production of ceramics and faiences from Uzège and trade with Catalonia.

Mots clés : Aigues-Mortes, géomorphologie, archéologie, port, Moyen Âge, Saint-Louis, fortification.

Keywords: Aigues-Mortes, geomorphology, archaeology, harbour, Middle Age, Saint-Louis, fortification.

\footnotetext{
a Université de Montpellier 3, UMR 220 Gouvernance Risque Environnement Développement-Route de Mende 34199 MONTPELLIER cedex 5. (tony. rey@univ-montp3.fr)

${ }^{b}$ Université d'Aix-Marseille, UMR 7298 Laboratoire d'Archéologie Médiévale et Moderne en Méditerranée - 5 rue du Château-de-l'Horloge, Maison méditerranéenne des sciences de l'Homme, 13094 AIX-EN-PROVENCE. (markeo@wanadoo.fr)

c Centre des monuments nationaux, Tours et Remparts d'Aigues-Mortes, Logis du Gouverneur, 30220 AIGUEs-MoRTES. (patrick.florencon@monumentsnationaux.fr)

'EPCC-CERP de Tautavel-Avenue Léon-Jean Grégory,66720 TAUTAVEL. (aslc@cerptautavel.com)
} 
Grâce aux découvertes archéologiques et aux sources historiques, les côtes méditerranéennes représentent une aire d'étude privilégiée pour étudier les interactions entre les changements environnementaux et l'évolution des sociétés.

La recherche géoarchéologique le long des côtes méditerranéennes françaises traite de divers thèmes. De nombreux auteurs ont étudié les anciens ports marins, lagunaires, fluvio-lagunaires, lesquels ont cessé leur activité en raison du colmatage sédimentaire, des variations du niveau de la mer, de la subsidence et des variations du trait de côte en lien avec la dynamique progradante des fleuves côtiers (Vella et al., 2005; Rey et al., 2009; Anthony et al., 2014).

Les reconstitutions paléogéographiques et environnementales croisées aux données archéologiques ont apporté de nouveaux éléments de compréhension dans l'étude des ports, le choix de la localisation, leur activité et abandon, les travaux nécessaires à leur pérennité, (Marriner et Morhange, 2007 et 2010; Bagan et al., 2010; Goiran et al., 2014; Sanchez et al., 2014; Morhange et al., 2015), et permettent aussi d'aborder la question des aléas et des risques côtiers (Morhange et al., 2010; Bony et al., 2014).

Le travail présenté concerne les anciens ports d'AiguesMortes dont la ville se situe aujourd'hui à plus de 5 kilomètres de la mer méditerranée. Cette cité royale fondée en 1240 par Louis IX roi de France lui permet d'avoir une assise politique et territoriale sur le littoral Méditerranéen. Cette histoire géostratégique associée à l'image pieuse de Louis IX (devenu Saint Louis) apparaissent bien cernées. En revanche, il existe peu de données lorsque l'on s'intéresse à la ville d'Aigues-Mortes, les ports et la plaine côtière. Les données textuelles et cartographiques historiques sont pauvres, tout comme les données géomorphologiques et archéologiques.

Pour tenter de répondre aux interrogations soulevées, un Projet Collectif de Recherche est conduit autour de la géoarchéologie des ports médiévaux d'Aigues-Mortes (coord. Tony Rey). Il ambitionne une meilleure compréhension des paléoenvironnements littoraux, des processus morphodynamiques côtiers, ainsi que des logiques d'organisation de l'espace portuaire et urbain.

Cet article présente les données morpho-sédimentaires inédites acquises au cours de la première campagne de carottages réalisée au pied du rempart de la cité. Les données sont croisées aux résultats d'une fouille archéologique conduite au pied du rempart d'Aigues-Mortes en 2011 (Markiewicz, 2011). Grâce aux techniques et méthodes géoarchéologiques, l'approche interdisciplinaire réalisée à haute résolution spatio-temporelle contribue à une meilleure connaissance de la cité portuaire d'Aigues-Mortes et de son environnement.

\section{ConteXte historiQue géNÉral}

En 1240, Louis IX lance une grande campagne de travaux non loin du littoral méditerranéen, à proximité d'un embarcadère rudimentaire que les textes mentionnent depuis 1231 environ (Florençon, 1996). La zone est encore presque vide d'hommes, en dehors des moines de l'abbaye de Psalmodi et de quelques pêcheurs qui exploitent la lagune. En arrière du rivage protégé de la mer par des cordons dunaires et un réseau de marais, le roi fait ériger une puissante tour ronde, la " grosse tour du roi », emblème de la dynastie. Son objectif est multiple :

- marquer de l'empreinte royale l'étroite fenêtre dont dispose le royaume de France sur la mer, entre les possessions du Saint Empire Romain Germanique qui englobent la Camargue à l'est, et celles du roi d'Aragon, qui s'avancent au-delà de Montpellier jusqu'aux limites de l'étang de Mauguio, à l'ouest;

- enchâsser un coin au milieu des terres du comte de Toulouse, qui demeure suspect dans ces derniers épisodes de la croisade contre les Albigeois;

- ouvrir enfin son domaine sur la Méditerranée, une mer qui connaît un essor économique formidable en ce début du XIII ${ }^{e}$ siècle, essor que le mouvement des croisades ne peut qu'accélérer.

Alors que le chantier des constructions royales avance rapidement, mettant au défi une région plutôt hostile (les marécages sont si présents dans le paysage qu'ils ont donné leur nom à la cité du roi, la "ville des eaux mortes »), Louis IX cherche à attirer et fixer une population qu'il veut nombreuse et particulièrement favorisée, en promulguant, dès 1246 , une charte de privilèges pour la ville et ses habitants d'une étonnante modernité : d'emblée la ville sera dotée d'un consulat, cadre administratif qui est partout ailleurs le fruit d'une longue lutte menée par le corps municipal.

Avant même de s'embarquer pour la Terre Sainte dans le port d'Aigues-Mortes (Florençon, 1996), Louis IX poursuit l'aménagement de cet espace en plaçant la ville au centre d'un réseau de voies de communication : profitant d'anciens cours d'eau atterris ou perçant de nouvelles voies artificielles, le roi place sa cité à proximité de cours d'eau (Rhône, Vidourle, Vistre) entre la Provence et le Languedoc : AiguesMortes, port méditerranéen, devient étape rhodanienne, exutoire naturel des foires de Champagne. Vers le nord et par la route, il fait du site l'avant port de la cité marchande de Nîmes.

Une population aux origines variées vient rapidement s'établir au pied du château royal. Pour la protéger aussi bien d'éventuelles attaques ennemies que des assauts des vents qui sur cette zone parfaitement plate déplace et accumule 
les sédiments, Louis IX lance en 1266 le projet de ceindre sa ville d'épaisses murailles. Mais il n'en connaîtra pas la réalisation : revenu à Aigues-Mortes pour s'embarquer une nouvelle fois pour la croisade, il meurt à Tunis en août 1270 .

\section{Des SourCes Peu abondantes}

Alors que les raisons qui ont poussé Louis IX à choisir Aigues-Mortes comme port méditerranéen semblent clairement identifiées, il est édifiant de constater les incertitudes et les mystères que recèlent la cité. Les trouvailles archéologiques apparaissent modestes face à l'imposante cité qui vient de traverser 800 ans d'Histoire sans grandes transformations. Nous retiendrons principalement la découverte en 1835 de tombes, situées en bordure de l'étang du Repausset, à 5 kilomètres d'Aigues-Mortes ${ }^{1}$. Associées à un ancien édifice disparu (l'hospice de Saint-Louis), les pierres tombales portant des épitaphes ont été datées du XIII ${ }^{\mathrm{e}}$ siècle (Mauzauric, 1910). La présence de ces sépultures de qualité doit être associée à un édifice d'importance en lien probable avec l'ancien site du port maritime mentionné dès 1231 $(\text { Aquae Mortuae) })^{2}$. Ce site, reconnu au XviII ${ }^{\mathrm{e}}$ siècle déjà, est reporté en 1778 sur la carte de Cassini (Provost, 1999). C'est à proximité, et en direction de la ville, que fut découvert dans le secteur de la Peyrade le tronçon de jetée. Construite en pierres à bossage, la construction rappelle en tous points les fortifications. Ce môle est traditionnellement interprété comme un soutènement ou digue. Cet aménagement est réalisé plus tardivement sous le règne de Philippe III.

Les mentions relevées de la ville médiévale d'AiguesMortes dès la fin du XII siècle n'ont donné lieu à aucune découverte illustrant la période. Ces mentions concernent l'activité de l'abbaye de Psalmodi, alors propriétaire d'un vaste territoire. Les chantiers (transports de pierres, sable et présence de maçons) sont connus sur le site dès 1240 . L'année 1246 marque le commencement du chantier royal avec la création du port défendu par un ouvrage puissant utilisé également comme phare ("la tour du seigneur roi des Français » telle qu'elle est désignée en 1249). En 1248, la ville est dotée d'une chaussée et de ponts permettant de l'atteindre. Dès cette date, les fortifications sont commencées et le port est relié à la ville par une route neuve. L'Inventaire Général des monuments et des richesses artistiques de France (1973) constitue le premier document de base pour l'étude des fortifications.

1. Le lieu dit Les Tombes est localisé sur la figure 1.

2. Archives municipales de Montpellier, Grand Thalamus, $\mathrm{f}^{\circ} 32 \mathrm{v}^{\circ}$, et Livre Noir, $f^{\circ} 41 \mathrm{v}^{\circ}$, éd. A. Germain, Histoire du commerce de Montpellier, I, Montpellier 1861, pièce justificative ${ }^{\circ}$ XII, p. 195-196.
D’une manière générale, le potentiel archéologique est resté longtemps insoupçonné. Nous pouvons établir ce même bilan au sujet des reconstitutions environnementales pour la période médiévale qui restent incertaines, empreintes d'incertitudes et de hiatus géochronologiques. Les textes et les cartes anciennes de C. Lenthéric (1876) ou de C.-F. Cassini (1778) n'apportent rien de plus de l'état du littoral, et certaines des conclusions avancées par J. Pagézy (1879) à la fin du xix siècle doivent être aujourd'hui repoussées. L'apport scientifique des cartes géologiques dressées par L'Homer (1987 et 1993) est incontestable, pourtant les extrapolations et les erreurs de localisation voilent la réalité paysagère. Enfin, les dernières études géohistoriques (Bellet et Florençon, 2001) et géoarchéologiques (Rey, 2006 et 2010; Markiewicz, 2011; Rey et Roure, 2015) conduites sur la cité d'Aigues-Mortes et sur la plaine occidentale du delta du Rhône s'attachent à combler certaines lacunes.

\section{Cadre géomorphologique}

La cité d'Aigues-Mortes se situe à l'extrémité occidentale de la plaine deltaïque du Rhône. La topographie est plane, les points hauts naturels sont quasi inexistants hormis quelques montilles. La formation de la plaine a été contrôlée par les apports sédimentaires, les courants marins et l'élévation relative du niveau marin (L'Homer 1987 et 1993; Provansal et al., 1995; Vella et al., 2005; Arnaud-Fassetta et al., 2010). Les grandes étapes de la formation de la plaine deltaïque sont aujourd'hui bien cernées même si les incertitudes apparaissent lorsque l'on travaille à plus haute résolution sur un espace ou sur une période précis. Plus à l'ouest, ce sont les dynamiques hydro-sédimentaires du Vistre et du Vidourle qui ont participé à l'extension de la plaine littorale. Le plus efficace dans cette construction est le Vidourle particulièrement actif à certaines époques (Berger et al., 2010). Pendant très longtemps le fleuve s'est déversé dans une lagune avant d'être canalisé jusqu’à la mer Méditerranée au XIX ${ }^{e}$ siècle. Le rôle du Vistre apparaît plus secondaire, moins puissant et moins tempétueux que le Vidourle. Il se perdait dans les marais à proximité de l'abbaye de Psalmodi (Py et Roure, 2002) puis a été canalisé vers d'autres canaux avant le $\mathrm{XvIII}^{\mathrm{e}}$ siècle. Une partie de cette histoire de l'environnement littoral peut se lire à partir des dépôts et des formes littorales et fluviales identifiées en surface (paléodunes, lagunes, étangs littoraux, paléochenaux). Aujourd'hui la cité d'Aigues-Mortes se situe dans une plaine relativement bien drainée et se positionne à environ 5,5 kilomètres de la côte (figure 1); une ambiance et un paysage assez éloignés de celui qui a prévalu au moment de sa fondation au XIII ${ }^{\mathrm{e}}$ siècle (Bellet et Florençon, 2001). 


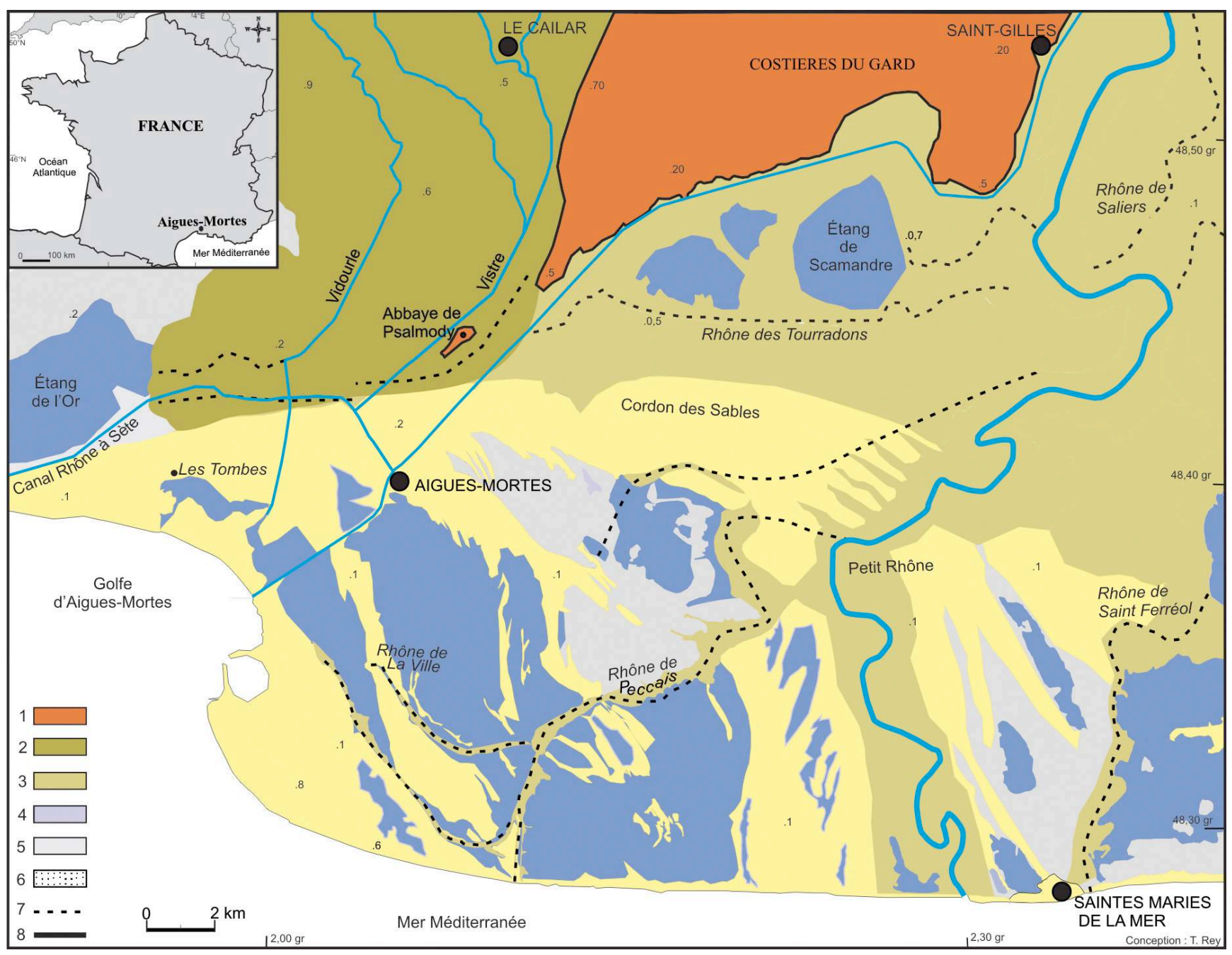

Figure 1 : (Voir planche couleur XX) Contexte géomorphologique du site d'Aigues-Mortes. 1 : Substrat pré-Holocène; 2 : Alluvions du Vistre et du Vidourle; 3 : Alluvions rhodaniennes; 4 : Étangs; 5 : zones humides; 6 : Cordons littoraux; 7 : Paléo-chenaux (Peccä̈) ; 8 : Chenaux actifs.

Figure 1: (See colour plate XX) The geomorphological context of the Aigues-Mortes medieval harbour. 1: pre-Holocene substrate; 2: the alluvial plain of Vistre and Vidourle channel; 3: the plain alluvial of the Rhone distributaries; 4: Ponds; 5: Wetlands; 6: Beach barriers; 7: Paleo-channels (Peccaï); 8: Active channels.

\section{Méthodologie}

Les travaux géoarchéologiques ont été conduits sur le rempart méridional car il est le seul à conserver dans sa maçonnerie des anneaux d'amarrage qui, fort de leur présence, suggèrent l'existence d'un embarcadère (figure 2).

La cité portuaire a été étudiée à partir d'une part, d'une fouille en tranchée (nommée S.02) au pied du rempart méridional aux abords de la Porte de la Marine de façon à obtenir la base des fondations de la cité et la stratigraphie avant et post-construction, et d'autre part, de plusieurs carottages face au même rempart dont deux - code
ARS 5 et ARS 6 - des plus significatifs des dynamiques environnementales sont présentés (figure 2). Les carottages ont été réalisés au carottier russe (UMR 5140 Plateforme ARPEGO), d'autres en raison des matériaux rencontrés ont été faits à la tarière. Les profondeurs atteintes au cours des carottages ont été variables, d'une manière générale et quel que soit l'outil utilisé, nous avons été bloqués par des sables marins compacts. Sur site ou en laboratoire, la stratigraphie de chaque carottage a été déterminée : texture, structure, couleur sur sédiments humides avec la charte de couleurs de sol Munsell et macrofaunes. Pour certains échantillons, la fraction sableuse a été tamisée et examinée sous une loupe 
Figure 2 : Le rempart sud de la cité fortifiée d'Aigues-Mortes; localisation de la fouille S.2 et des carottages ARS 5 et ARS 6. Figure 2: The fortress of AiguesMortes; location of the archeological core S.O2 and the core drilling ARS 5 et ARS 6.

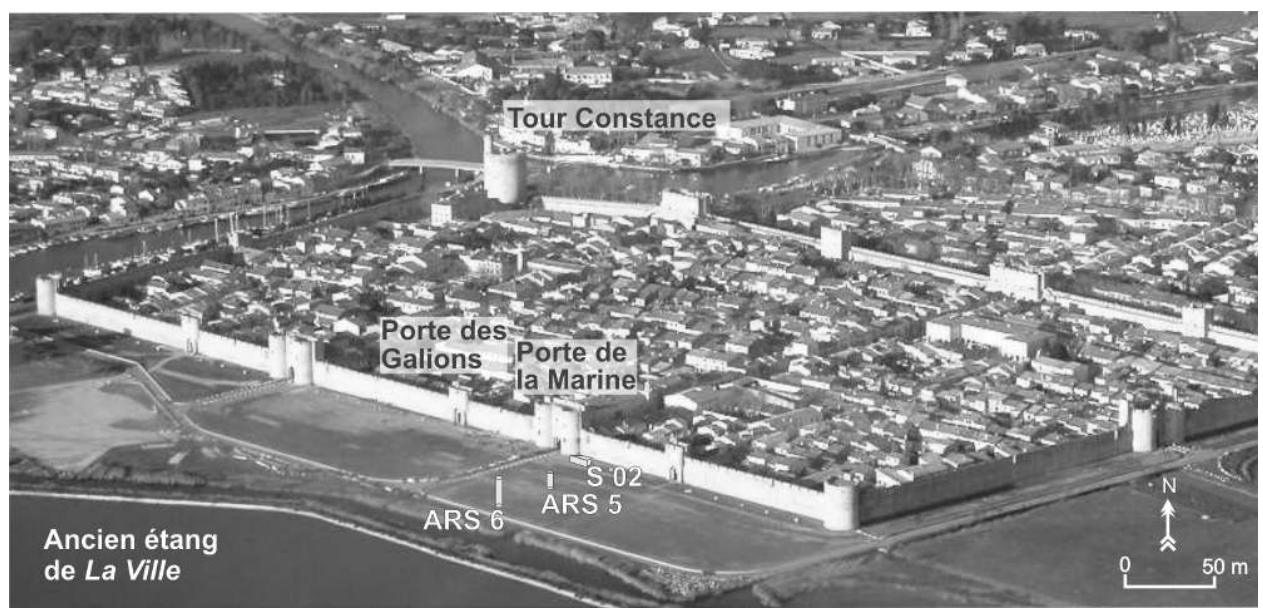

binoculaire. Les échantillons ont été prélevés pour d'autres analyses en laboratoire (granulométrie et datation $\mathrm{C}^{14}$; palynologie et ostracologie en cours). Pour chaque carottage, la succession des environnements de dépôts est illustrée en identifiant les faciès sédimentaires.

Le cadre géochronologique a été fourni par des datations au radiocarbone conventionnelles et AMS (tab. 1). La calibration des dates a été réalisée avec le programme OxCal v.4.2.3 développé par Bronk R. (2013) en se basant sur les données atmosphériques de IntCal13 (Reimer et al., 2009). Concernant la fouille S.02, la datation des niveaux s'est essentiellement appuyée sur des tessons de céramique et d'autres restes archéologiques.

\section{RÉSULTATS DE LA FOUILLE ARCHÉOLOGIQUE FAITE À la PorTe de la Marine (SONDAge S.02)}

La fouille s'est étendue sur une surface inférieure à $2 \mathrm{~m}^{2}$ et a atteint 2,4 mètres de profondeur. Les données mettent en évidence une succession de niveaux d'occupation du XIII ${ }^{\mathrm{e}}$ au XVIII ${ }^{\mathrm{e}}$ siècle (figure 3 ).

\section{Les contextes tardifs}

Les données illustrent le rechaussement général réalisé au $\mathrm{XVIII}^{\mathrm{e}}$ siècle, ainsi que la conservation de niveaux médiévaux liés aux fondations non massives.

L'aspect actuel de ce secteur méridional transformé en vaste esplanade résulte de rechaussements tardifs du terrain (S.02/03 et S.02/04). Les apports de surface observés sur deux couches fournissent de la terre sur une épaisseur de 1 mètre. Ces niveaux doivent être vraisemblablement rattachés aux campagnes ultimes de nivellement d'époque contemporaine. Le terrain légèrement plus élevé contribue à expliquer, au sud de la ville, un enfouissement plus important des contextes archéologiques anciens d'époque médiévale.

L'occupation du XVIII ${ }^{\mathrm{e}}$ siècle est formellement attestée et matérialisée par un niveau de sol chaulé (S.02/05) qui démontre la volonté d'assainir les espaces extérieurs, tout en

\begin{tabular}{|c|c|c|c|c|c|}
\hline Code échantillon & $\begin{array}{c}\text { Code } \\
\text { laboratoire }\end{array}$ & $\begin{array}{l}\text { AMS Age C14 } \\
\text { (BP) }\end{array}$ & $\begin{array}{c}\text { Âge calibré } \\
(2 \sigma)\end{array}$ & $\begin{array}{c}\text { Âge calibré } \\
(\text { Cal BC-AD) }(2 \sigma)\end{array}$ & Matériel \\
\hline GdA-3627 & Ars 6-235 & $625 \pm 25$ & $1290-1400 \mathrm{AD}$ & $1345 \pm 55$ & Bois \\
\hline GdA-3625 & Ars 6-310 & $800 \pm 20$ & $1210-1270 \mathrm{AD}$ & $1240 \pm 30$ & Dent \\
\hline GdA-3625 & Ars 6-440 & $4970 \pm 35$ & $3905-3660 \mathrm{BC}$ & $3780 \pm 120$ & Bois \\
\hline GdA-3625 & Ars 6-620 & $910 \pm 25$ & $1035-1185 \mathrm{AD}$ & $1110 \pm 75$ & Coquille \\
\hline GdA-3629 & Ars 5-175 & $850 \pm 20$ & $1160-1250 \mathrm{AD}$ & $1205 \pm 45$ & Bois \\
\hline \multirow[t]{2}{*}{ GdA-3628 } & Ars 5-183 & $1070 \pm 25$ & $900-1020 \mathrm{AD}$ & $960 \pm 60$ & Bois \\
\hline & & $\begin{array}{c}\text { Âge C14 } \\
\text { conventionnel }\end{array}$ & & & \\
\hline Beta 379229 & Pieu Sud AM1 & $540 \pm 30 \mathrm{BP}$ & $1320-1435 \mathrm{AD}$ & $1380 \pm 60$ & Bois \\
\hline
\end{tabular}

Tableau 1 : Les datations radiocarbone réalisées sur le site d'Aigues-Mortes.

Table 1: The radiocarbon dating carried out on the site of Aigues-Mortes. 


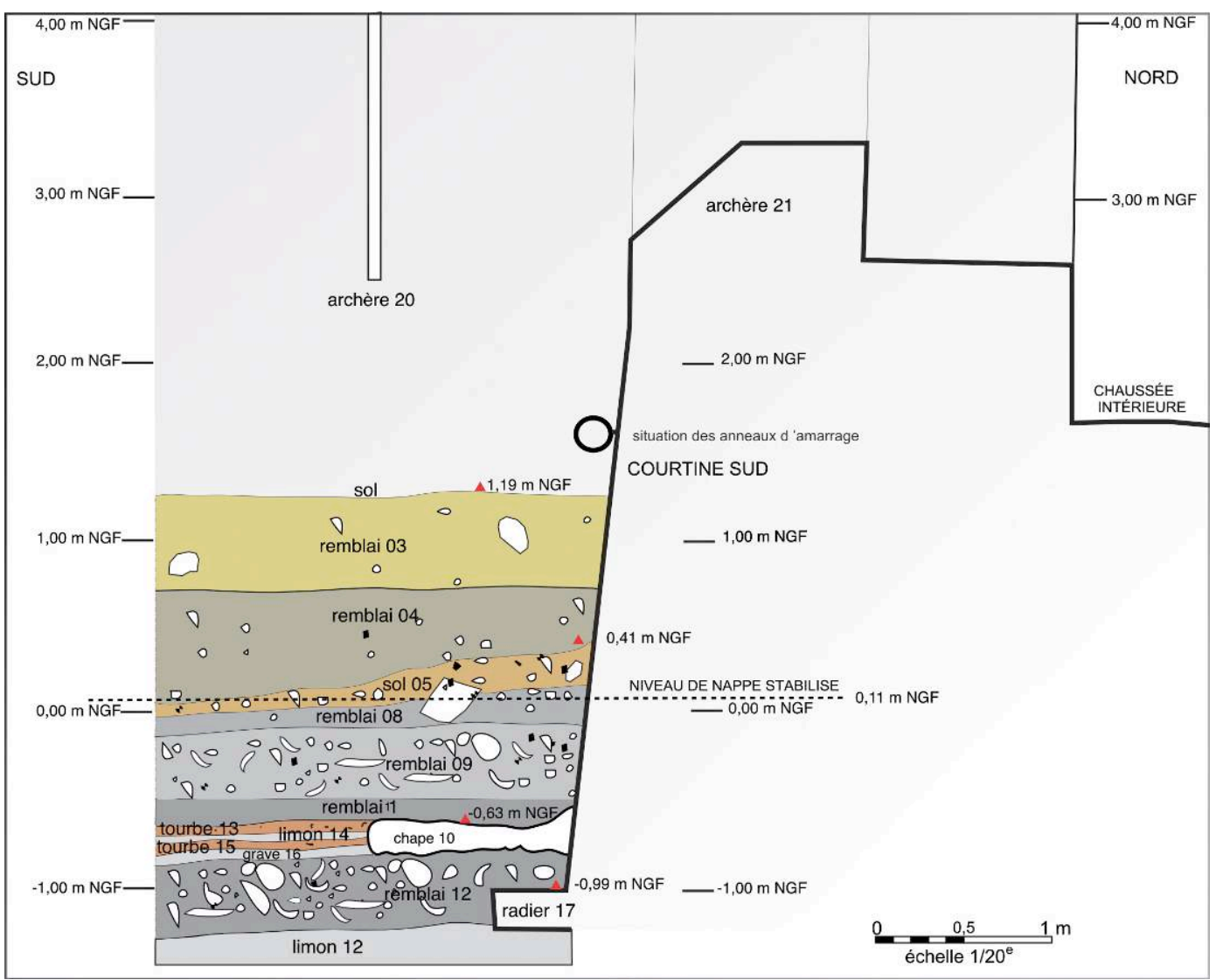

Figure 3 : (Voir planche couleur XXI) Interprétation chronostratigraphique des niveaux de la fouille S.02.

Figure 3: (See colour plate XXI) Chronostratigraphic interpretation of the archaeological core S.02. permettant d'y circuler (figure 3). Quelques blocs de pierre abandonnés sur place suggèrent le stockage de matériaux, destinés probablement à quelque restauration. Implanté à cette altitude de 0,40 m NGF, le sol était ainsi préservé de la nappe. Les observations archéologiques conduisent à envisager un niveau de nappe situé plus bas au cours des siècles passés. Ce niveau stabilisé de la nappe coïncide en sous-sol avec le remblai de démolition d'époque moderne (S.02/09).

Ces observations corroborent les données acquises à Marseille où le niveau de la mer stationne à $-12 \mathrm{~cm}$ NGF vers 1660 (Morhange et al., 2001).

Par sa composition, cette recharge bien datée par le mobilier céramique exprime la volonté d'exhausser le niveau du terrain et de repousser les berges de l'ancien étang vers le sud. Si l'on se fie à la cartographie ancienne, ce programme pourrait être daté des décennies 1750-1770.

\section{Les contextes médiévaux et leur datation}

Les contextes médiévaux conservés au contact de la courtine et de la Porte de la Marine sont atteints à 1,60 m par rapport au sol actuel. Un amas de mortier de chaux contenant galets et débris en terre cuite dégagé à $-0,63 \mathrm{~m}$ NGF révèle une aire de travail $(S .02 / 10)$. Ce témoignage d'une activité au pied des constructions est observé, sous une couche organique (S.02/11), au niveau d'une recharge de matériaux (S.02/12) qui démontre la nécessité dans ce secteur de surélever momentanément le terrain, sujet à des fluctuations hydrologiques (figure 4). Le remblai (S.02/12) fournit des indices de datation qui renvoient à une phase fin $\mathrm{XIII}^{\mathrm{e}} / \mathrm{XIV}^{\mathrm{e}}$ siècle. Le lot se répartit en quatre catégories : la céramique de stockage, les faïences, la céramique grise fine et les productions de l'Uzège.

- Le premier groupe est représenté par des fragments à paroi épaisse ou plus fine. Les pièces fragmentées annoncent des contenants volumineux dont le diamètre du corps avoisine les $52 \mathrm{~cm}$. Les vases en céramique calcaire à paroi fine de couleur rose sont représentés par quatre tessons dont l'un est un bord de $15 \mathrm{~cm}$ de diamètre. Trois fragments de panses en pâte réfractaire glaçurée désignent les ateliers de l'Uzège.

- Les faïences polychromes appartiennent à quatre formes différentes, deux plats à marli et trois coupes représentatifs des productions catalanes (figure 5). Les plats à marli et paroi fine, similaires dans leur forme générale, se distinguent par l'inclinaison mesurée du marli (figure 6). D'un diamètre de 28 et $32 \mathrm{~cm}$, elles sont en pâte calcaire rouge et l'une d'elles présente une cuisson mixte marquée dans l'épaisseur de la pâte par une fine trace noire. Le marli a une largeur 
Figure 4 : Le dégagement en cours du remblai inférieur médiéval (cliché Ch. Markiewicz).

Figure 4: The Medieval inferior backfill.

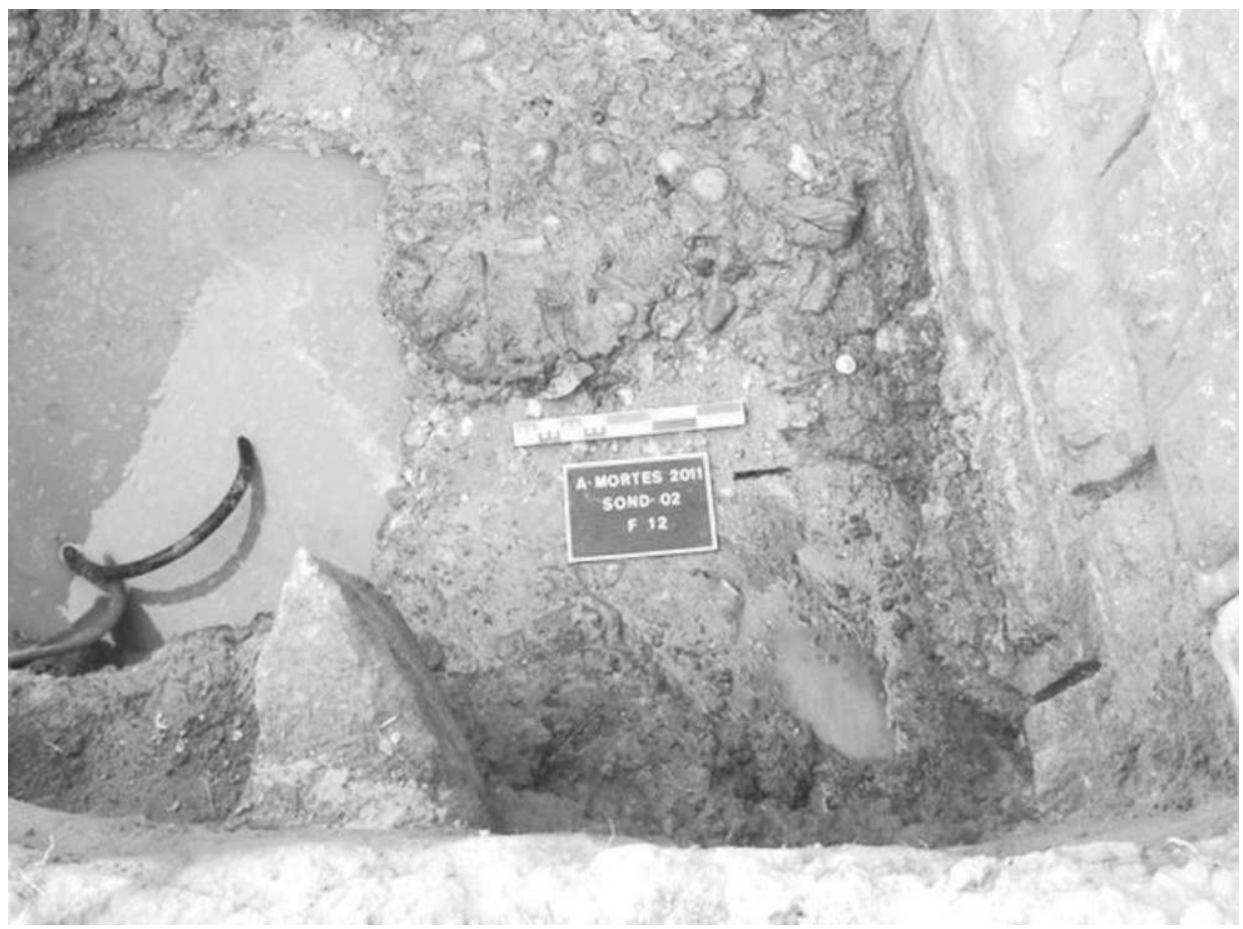

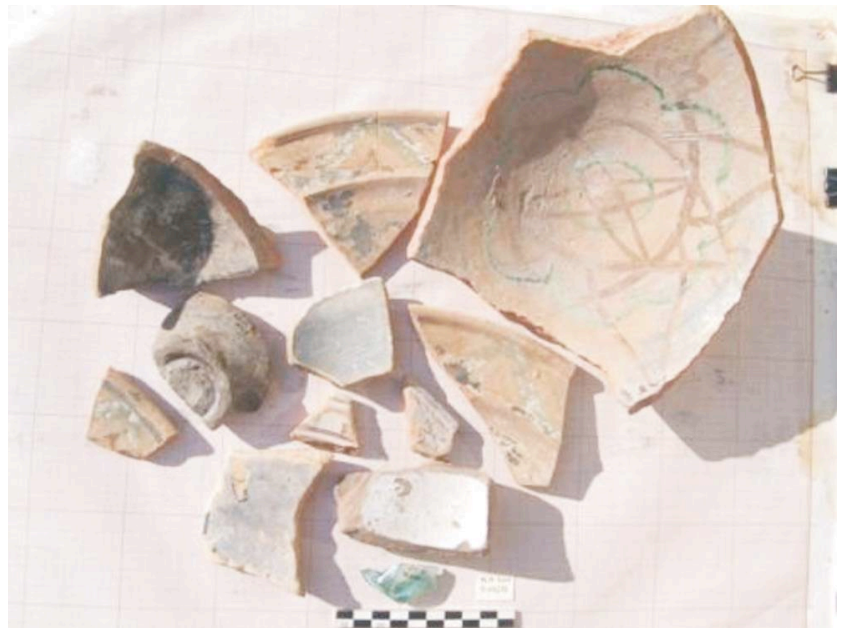

Figure 5 : (Voir planche couleur XXI) Les faïences extraites du contexte S.02/12 (cliché Ch. Markiewicz).

Figure 5: (See colour plate XXI) The faience vessels from the level S.02/12.

de 4,3-4,4 cm et le bord prolonge à l'extérieur le profil de la forme. À l'intérieur, il marque un léger bourrelet à la naissance du marli. La limite du marli à l'intérieur du plat est marquée par un petit ressaut saillant sur surmonte la panse légèrement incurvée. Le décor intérieur, très érodé, représente sur les deux formes des séries de $\mathrm{V}$ de couleur verte reliés entre eux et à l'intérieur desquels s'inscrivent des triangles de couleur brune aux lignes courbes. Si l'on excepte les formes légèrement différentes des deux coupes,

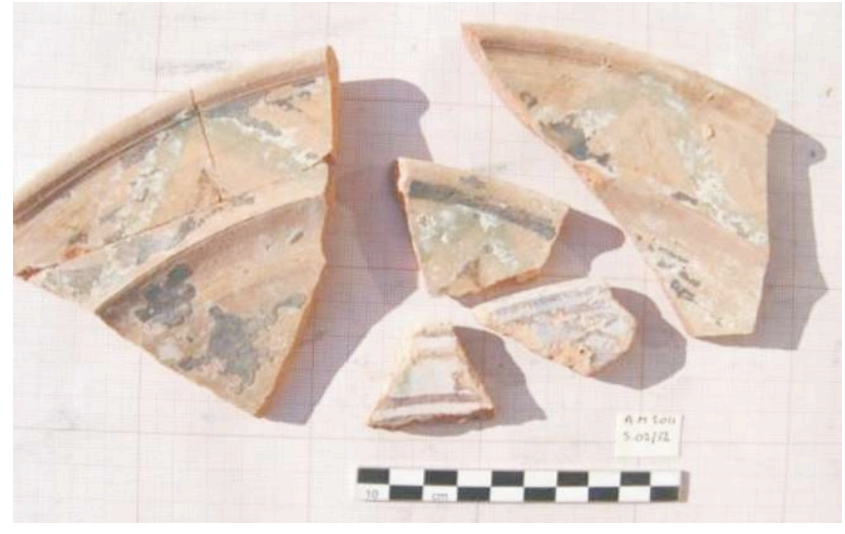

Figure 6 : (Voir planche couleur XXI) Les coupes d'importation extraites du contexte S.02/12 (cliché Ch. Markiewicz).

Figure 6: (See colour plate XXI) The faience vessels import from the level S.02/12.

le décor identique suggère en revanche une production réalisée dans un même atelier. Les parties conservées des panses conservent des lignes brunes entourant vraisemblablement un motif central. La coupe à paroi plus épaisse appartient à une forme plus volumineuse. Elle est brisée horizontalement dans sa moitié, elle a un diamètre $20 \mathrm{~cm}$ qui indique une ouverture de $30 \mathrm{~cm}$ environ. Cette forme incurvée repose sur un pied annulaire assez massif qui atteint un diamètre extérieur de 11,3 cm. La partie intérieure conserve également un décor lessivé complexe dominé par un polylobe 


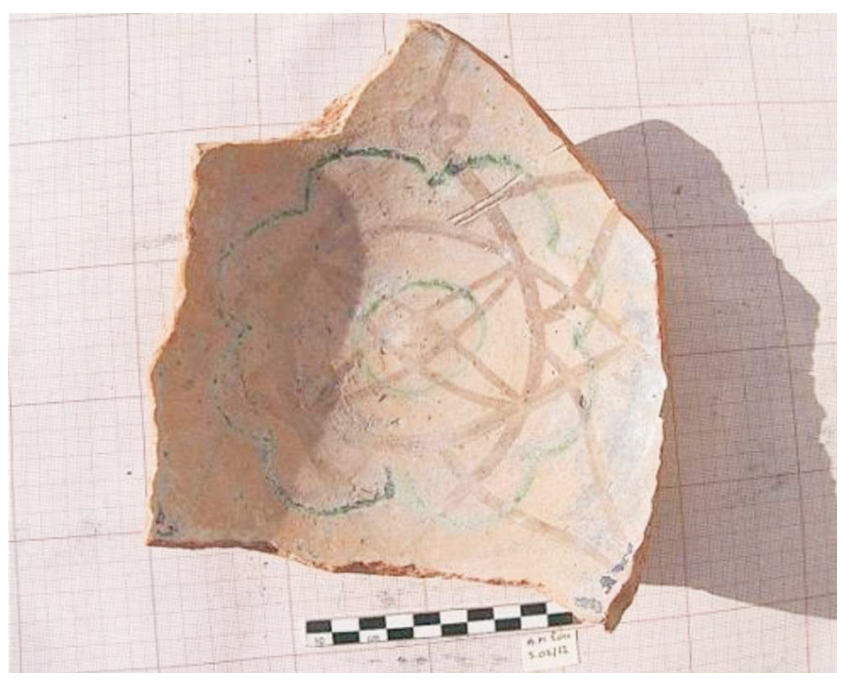

Figure 7 : (Voir planche couleur XXII) La coupe catalane à paroi épaisse extraite du contexte S.02/12 (cliché Ch. Markiewicz). Figure 7: (See colour plate XXII) The Catalan ceramic from the level S.02/12 (cliché Ch. Markiewicz).

central de couleur verte à huit pétales. Ce motif principal se superpose à quatre navettes brunes croisées en rosace qui s'achèvent par une figuration végétale stylisée en forme de trèfle. Au contact des navettes, un décor en forme de flamme comble le vide. La partie centrale de la coupe est occupée par deux navettes plus fines de couleur brune dont la jonction s'inscrit dans un cercle vert. L'ensemble est conçu comme une composition géométrique étoilée, motif fréquent dans les productions catalanes et reconnues en de nombreuses occasions dans les contextes datés de la fin du XIII ${ }^{e}$ et début du XIv ${ }^{\mathrm{e}}$ siècle.

Une série de fragments de petite taille permettent d'identifier un bol monochrome blanc ainsi que plusieurs autres coupes catalanes (figure 7). Notons, enfin, à l'intérieur de ce contexte (S.02/12), la présence d'un fragment de verre épais à décor étoilé moulé.

\section{La question des fondations}

À la base des constructions, l'élévation en moellons à bossage prend appui sur une structure saillante qui marque la base des constructions. À ce niveau, les assises intégrées à la partie talutée sont traitées à l'identique des autres moellons utilisés dans l'élévation. Ils présentent toutefois sur trois assises des marques d'érosion par l'eau qui donnent aux moellons un aspect fruste. Cet état d'usure peut expliquer le rechaussement rapide, réalisé au XIv siècle à l'aide du remblai de démolition (S.02/12) qui supporte l'aire de travail (S.02/10).
La base des constructions dévoile une structure de dalles étroites ou de blocs qui sert d'appui à la première assise de moellons (figure 8 ). Ces pièces présentent une largeur variable, comprise entre 0,30 et $0,40 \mathrm{~m}$ et intègre sous la courtine un bloc plus étroit intercalé. L'épaisseur régulière est de 0,24 m. L'ensemble forme un radier de pose servant à asseoir la base des constructions. Dans des conditions techniques difficiles, un repérage sous les dalles a été tenté manuellement. Un vide important est reconnu horizontalement sous les blocs qui se prolongent à l'aplomb de l'élévation. Cette observation permet d'exclure un type de fondation massive et débordante formée par une semelle maçonnée puissante. De toute évidence, le radier de pose doit être associé à une superstructure située sous les constructions et dont la constitution nous échappe. Placé dans l'épaisseur du limon sablonneux (S.02/18), ce système suggère une architecture porteuse utilisant le bois. Ce mode de construction renvoie aux fragments de bois extraits, hors stratigraphie, des couches profondes à l'aide du tractopelle, parmi lesquels figure une tête de pieu taillé en pointe (figure 9), ainsi qu'une cheville en bois de génévrier. La partie du pieu incomplet a une longueur de $50 \mathrm{~cm}$ pour un diamètre maximal de $13,5 \mathrm{~cm}$ qui se réduit en pointe. Des terenidae (mollusques bivalves appelés tarets) ont tronqué le pieu dans sa partie sommitale. Ces vers sont connus pour causer de très fortes dégradations aux installations portuaires et aux constructions navales (Tuente et al., 2002). Analysées par Lucie $\mathrm{Chabal}^{3}$, les pièces ont révélé pour le pieu une variété de conifère (Pinus halepensis : pin d'Alep ou Pinus Pinea : pin pignon). Il s'agit d'un bois indigène en Languedoc (Chabal, 1997). Parfois très dense, il est utilisé en menuiserie ou pour la construction navale ou comme bois d'œuvre, mobilier ou bois de construction (Chabal, 2012). Le bois a été daté 540+/-30 ans BP (Beta-379229), soit $1320-1435$ cal AD ce que confortent les niveaux archéologiques étudiés dans le sondage 02 .

\section{RÉSULTATS DES CAROTTAGES SÉDIMENTAIRES - Site Aigues-MorTes}

\section{Analyse du carottage ARS 5}

Le carottage ARS 5 ( $\left.4^{\circ} 11^{\prime} 29^{\prime \prime} .5 \mathrm{E}-43^{\circ} 33^{\prime} 51^{\prime \prime} .2 \mathrm{~N}\right)$ a été réalisé à 5,6 kilomètres du rivage actuel, et à 5 mètres du pied du rempart sud. Le carottage a atteint la profondeur de 4 mètres, arrêté par des sables trop compacts. La séquence présente 5 grandes unités stratigraphiques: (1) des sables de

3. Centre de Bio-Archéologie et d'Ecologie de Montpellier, chargée de recherche au CNRS-UMR 5059. 

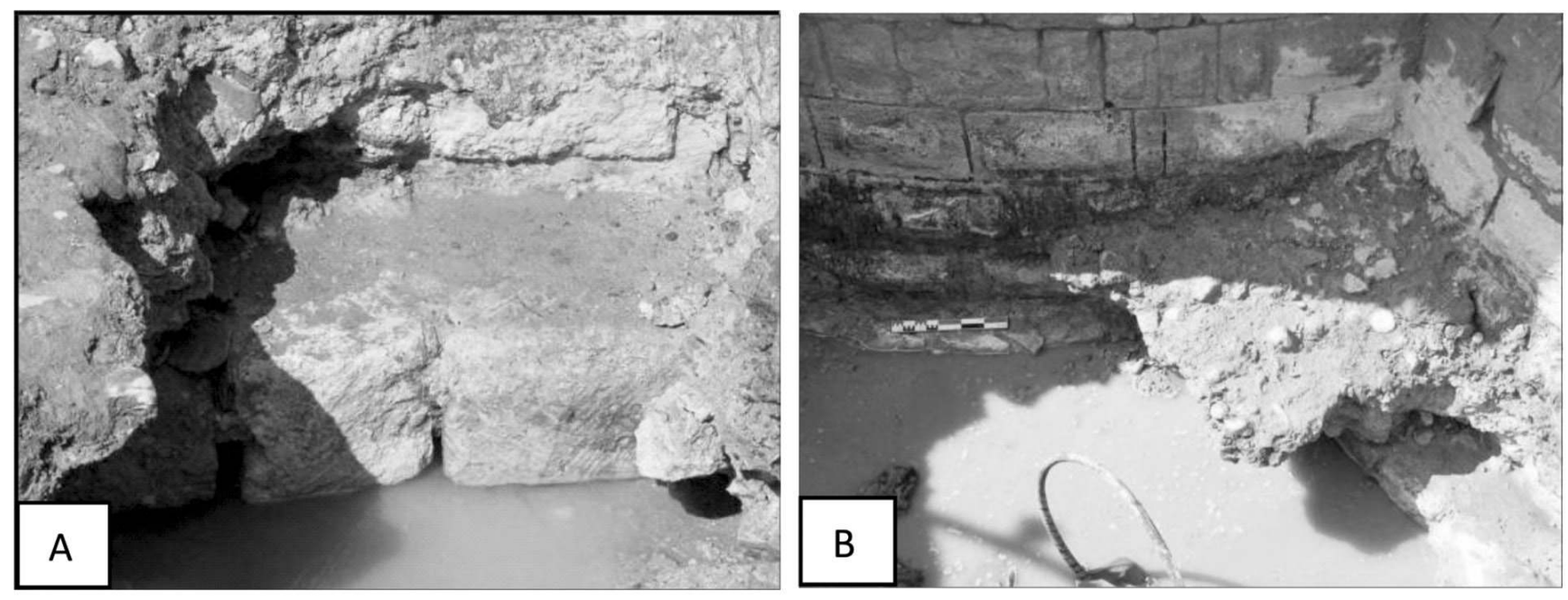

Figure 8 : L'apparition du radier de dalles à la base des constructions : A : la courtine, B : la tour (cliché Ch. Markiewicz).

Figure 8: The base of the fortification; $A$ : the wall between the towers; $B$ : the tower.

Figure 9 : Le pieu extrait hors stratigraphie (cliché T. Rey).

Figure 9: The pointed stake extract outside the stratigraphy S.02.

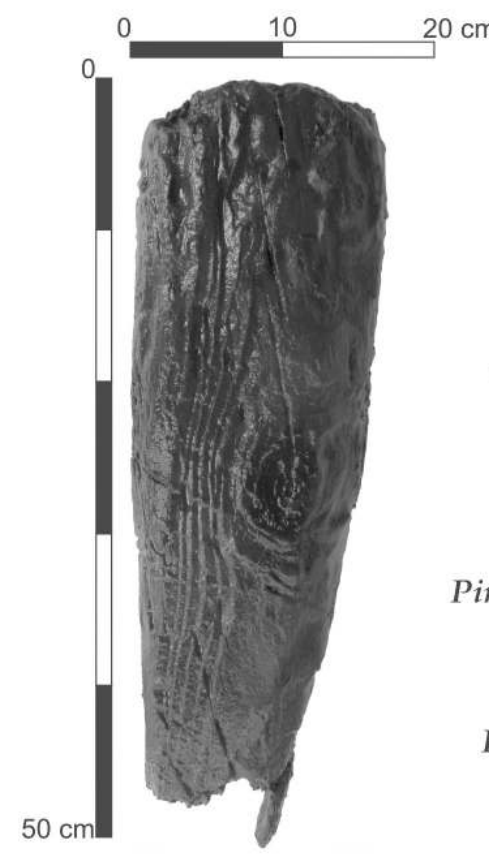

Pinus halepensis (pin d'Alep)

ou

Pinus Pinea (pin pignon) plage; (2 et 4) des limons organiques à ambiance marécageuse; et (3 et 5) des niveaux de remblais (figure 10).

- À la base de la séquence présentée, l'unité sédimentaire 1 (400-200 cm) se caractérise par des sables fins, azoïques, bien triés, de coloration claire (10 YR 5/8). Cette unité caractérise des sables marins dans un milieu relativement bien ouvert.

- L'unité 2 (200-185 cm) est composée de sédiments limoneux et organiques, de coloration sombre (5Y 3/2), riches en coquilles et fibres végétales. Quelques rares passées sableuses sont observables. Les données sédimentologiques évoquent une ambiance marécageuse. Au sommet de l'unité, le prélèvement (ARS 5-183) a été daté $1070 \pm 25$ ans BP (GdA-3628) soit $960 \pm 60$ cal AD.

- Les dépôts de l'unité $3(185-162 \mathrm{~cm})$ sont grossiers et hétérogènes. Les sables moyens à grossiers de couleur gris sombre (2,5 Y 4/0) s'accompagnent de nombreux gastéropodes lagunaires, de petits galets roulés $(1 \mathrm{~cm})$ et riches en débris végétaux. Les dépôts sont très hétérogènes, ils traduisent un remblai (sol d'occupation?). Au sommet de l'unité, le prélèvement (ARS 5-175) a été daté $850 \pm 20$ ans BP (GdA-3629) soit $1205 \pm 45$ cal AD.

- L'unité 4 (162-130 cm) est formée par l'accumulation de limons fins de coloration sombre (5Y 3/1), riches en 


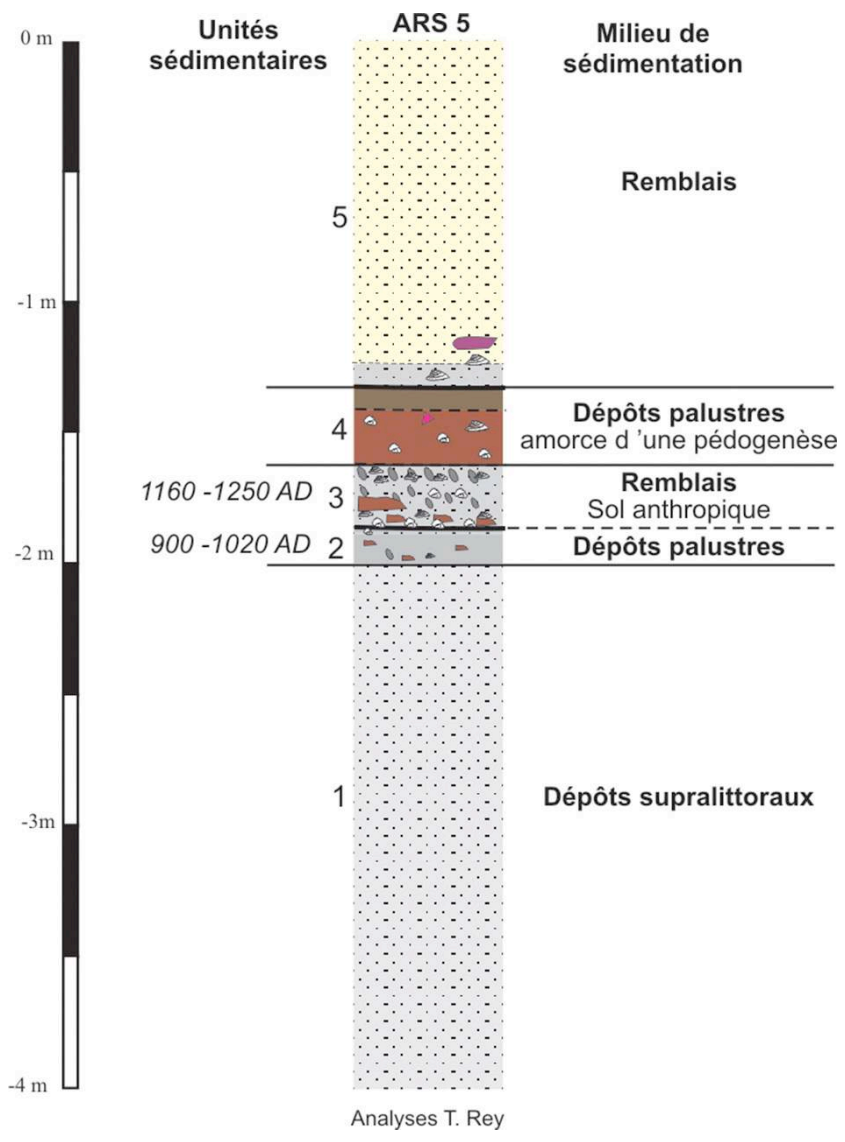

Figure 10 : (Voir planche couleur) Stratigraphie des carottes sédimentaires ARS 5 (site Aigues-Mortes).

Figure 10: (See colour plate) Core stratigraphy from site ARS 5 AiguesMortes.

coquilles de milieu palustre (Valvata sp. et Bythynia sp.) et enrichis en matière organique et fibres végétales indéterminées. Les données évoquent une amorce de pédogenèses humiferes dans une ambiance marécageuse.

- Au sommet de la carotte, l'unité $5(130-0 \mathrm{~cm})$ se caractérise par des sables fins, assez homogènes, de couleur jaune (5Y 5/6). La base de ces dépôts présente des morceaux de mortiers $(4 \mathrm{~cm})$.

Les analyses mettent en évidence deux environnements de dépôts : le premier un environnement marin dont la formation est antérieure à $960 \pm 60 \mathrm{cal} \mathrm{AD}$ (GdA-3628). Puis les dépôts assez hétérogènes et très organiques suggèrent une sédimentation terrigène lente en milieu palustre, lesquels sont associés à une succession de remblaiements aux abords du rempart afin de rechausser un terrain marqué par une forte hydromorphie. Le sommet de la séquence correspond à des remblaiements modernes (dépôts issus du creusement moderne du canal). Ces données sédimentaires sont en adéquation avec les données archéologiques (S.02).

\section{Analyse du carottage ARS 6}

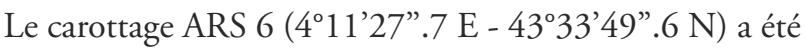
réalisé à 60 mètres du rempart sud, dans l'alignement du carottage ARS 5. Il a atteint la profondeur de 7,7 mètres sous la surface, arrêté par des sables fins très compacts. La séquence présente 3 principales unités stratigraphiques : (1) des sables infralittoraux; (2) des dépôts portuaires et (3) des dépôts palustres et (4) des remblais (figure 11).

- À la base de la séquence présentée, l'unité 1 (710$400 \mathrm{~cm}$ ) est composée principalement d'un sédiment sableux, très fin, de couleur gris foncé $(5 \mathrm{Y} 5 / 1)$ et pauvre en coquilles (une valve de cardium à $620 \mathrm{~cm}$ et une coquille de telline à $447 \mathrm{~cm}$ ). La fraction sableuse décroit progressivement vers le sommet de l'unité. Deux lits de sédiments plus grossiers et hétérogènes s'intercalent dans l'unité. Le premier, à $570 \mathrm{~cm}$, présente des galets de tailles variables (taille : $6 \mathrm{~cm}$ ) ainsi que des fragments de charbons. Le second lit $(460 \mathrm{~cm})$ contient des sables fins limoneux dans lesquels s'inscrivent des galets (taille : $3 \mathrm{~cm}$ ). L'unité 1 est interprétée comme un faciès marin notamment de l'étage infralittoral où au moins deux évènements de haute énergie (tempête) sont enregistrés. Deux datations au radiocarbone ont été réalisées sur les prélèvements ARS 6-440 et ARS 6-620 qui ont été datés $4970 \pm 35$ ans BP (GdA-3625) et $910 \pm 25$ ans BP (GdA-3625) soit $3780 \pm 120$ cal BC et $1110 \pm 75$ cal AD. L'inversion chronologique est discutée en synthèse.

L'unité 2 se subdivise en 3 grandes phases d'évolution:

- L'unité 2.a (400-310 cm) est formée par l'accumulation de limons sableux gris (5Y 5/1). Plusieurs restes archéologiques et faunistiques sont observés : un tesson vernis, des scories et probablement des déchets alimentaires provenant des activités des habitants du port : un murex brandaris non usé par l'action de la houle, une dent et des os de crâne appartenant à de jeunes caprins ${ }^{4}$. La transition avec le faciès sous-jacent est assez brutale indiquant une transformation rapide du milieu. Le sommet de la sous unité $2 . a$ a été daté à $800 \pm 20$ ans $\mathrm{BP}$ soit $1240 \pm 30 \mathrm{cal} \mathrm{AD}(\mathrm{GdA}-3625)$.

- L'unité 2.b (310-221 cm) est composée de limons plus fins, de couleur gris foncé ( $5 \mathrm{Y} 4 / 2)$, riches en coquilles lagunaires de milieu saumâtre (Cerastoderma et Loripes Lacteus), notamment des bivalves entiers et en position de vie. L'unité 2.b a été datée $625 \pm 25$ ans BP soit $1345 \pm 55$ cal AD (GdA3627).

- L'unité 2.c $(221-178 \mathrm{~cm})$ contient un sédiment limoneux gris sombre $(5 \mathrm{Y} 3 / 2)$, très organique notamment sous la forme de fibres végétales. Plusieurs lits limono-sableux

4. Détermination faite par Armelle Gardeisen, Archéozoologue, UMR 5140 du CNRS «Archéologie des sociétés méditerranéennes ». 


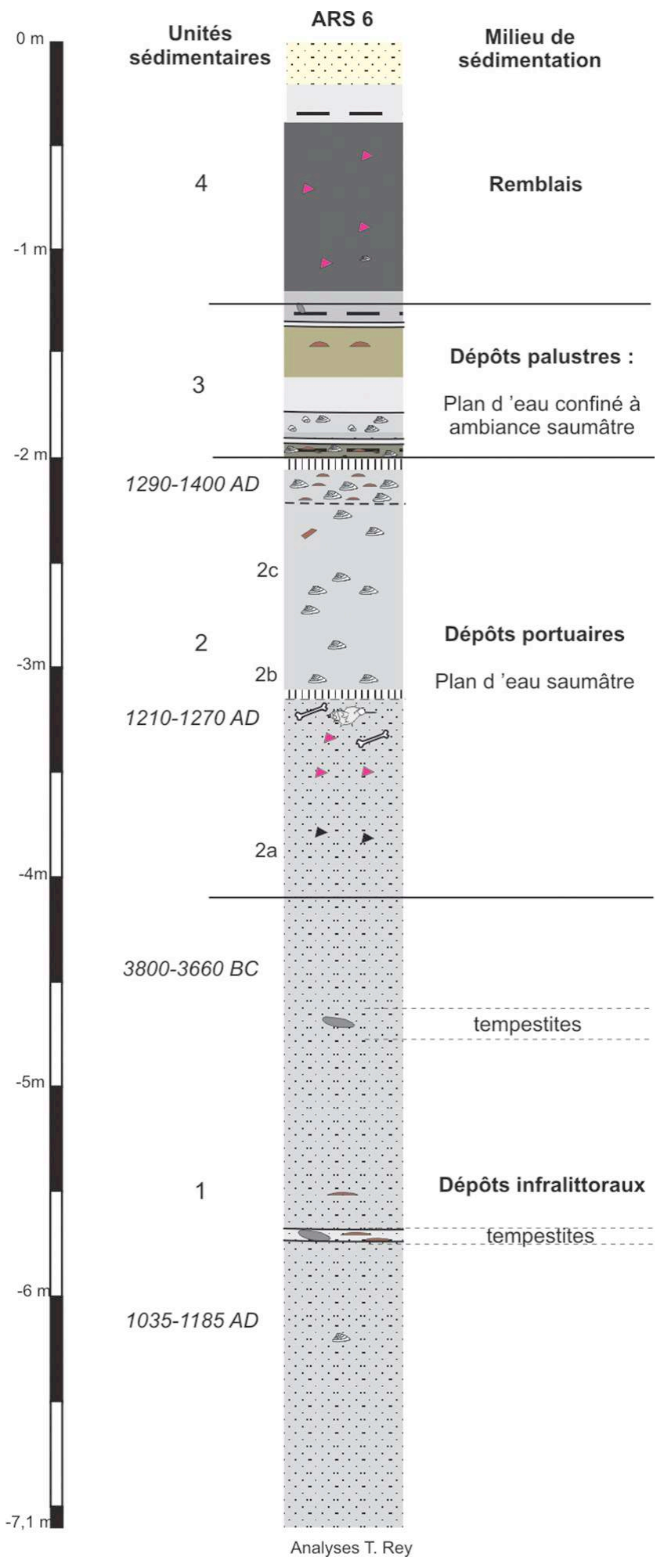

Figure 11 : (Voir planche couleur XXII) Stratigraphie des carottes sédimentaires ARS 6 (site Aigues-Mortes).

Figure 11: (See colour plate XXII) Core stratigraphy from site ARS 6 Aigues-Mortes. s'intercalent dans cette sous-unité $(192 \mathrm{~cm}$ et $188 \mathrm{~cm})$. Les bivalves de type Cardium abondent et sont associés dans la partie supérieure de l'unité à Hydrobia sp. Les données malacologiques et l'apparition d'espèces d'herbiers en sommet d'unité signalent une évolution du milieu vers un environnement de type lagunaire.

- L'unité $3(178-121 \mathrm{~cm})$ se caractérise par des limons de plus en plus fins, de coloration claire (5Y 5/1 à 5Y 5/4), à de rares coquilles et pauvres en débris végétaux. De la matière organique sous forme diffuse apparait vers $160 \mathrm{~cm}$ et $146 \mathrm{~cm}$. Les données sédimentologiques traduisent le confinement du milieu.

- Au sommet de la carotte, l'unité $4(121-0 \mathrm{~cm})$ montre une alternance entre de l'argile de coloration grise claire associée à des cailloux anguleux (121-110 cm) et des limons noirâtres $(110-40 \mathrm{~cm}$ ) chargés en restes anthropiques (fil de fer, tesson, verre) et de l'argile de coloration claire $(40-20 \mathrm{~cm})$ et enfin des sables jaunes (20-0). Ces niveaux très perturbés et pollués traduisent plusieurs niveaux de remblais.

\section{SYNTHÈSE GÉNÉRALE ET CONCLUSION}

\section{Aigues-Mortes : un port lagunaire aux ambiances palustres}

Les données géoarchéologiques confirment l'existence d'un milieu marin antérieur à la construction des murs d'enceinte de la cité. Ce milieu marin évolue ensuite vers un environnement lagunaire et c'est dans ce contexte que la cité et le port d'Aigues-Mortes vont prendre place. Les eaux de la lagune n'atteindraient pas le pied du rempart ${ }^{5}$ (comme le voulait la tradition à la vue des anneaux d'amarrage sur le mur du rempart). La ligne de ce rivage se situerait à environ 20 mètres du rempart sud. Pour accéder à cette rive sans trop de contraintes, plusieurs remblais ont été réalisés pour rechausser le terrain. La lagune a alors constitué le bassin portuaire naturel de la cité dont la profondeur aurait pu atteindre deux mètres si l'on tient compte d'une part des dépôts portuaires et d'autre part du calage chronologique des dépôts datés entre 1210 et 1400 AD (ARS 6 Unité 2). Une donnée qui doit être nuancée compte tenu de la nature variable des fonds lagunaires mais aussi des actions de dragage réalisées au XIII ${ }^{\mathrm{e}}$ siècle. Ces dragages constituent d'ailleurs un bon argument pour expliquer l'inversion chronologique présente dans le carottage ARS 6. Cette inversion pourrait également être liée au mode de prélèvement des échantillons et à la possibilité de pollution au cours de

5. L'étude ostracodologique précisera cet état ultérieurement. 
la remontée de l'outil. Cependant, l'existence d'inversions chronologiques rpeut aussi témoigner de la réalisation de dragages du port (Marriner et Morhange, 2006; Morhange et Marriner, 2009). Dès 1272, moins d'un demi siècle après la fondation de la cité portuaire, il est fait mention de curer le port (Pagezy, 1879). En 1278, sous le règne de Philippe III le Hardi, le port de la Peyrade est fondé à 5 kilomètres plus au sud de la cité, il devient alors le port principal avec une entrée protégée par une longue digue encore visible aujourd'hui. En 1289, le sénéchal de Beaucaire prévoyait un dragage régulier du port dont le coût avoisinait 1000 livres/ $\mathrm{an}^{6}$.

Sous le règne de Louis IX, le port lagunaire se comble rapidement exhaussant les fonds de près de $85 \mathrm{~cm}$ de sédiments entre le XIII ${ }^{\mathrm{e}}$ et le XIV siècle. Ces taux ne doivent pas surprendre car, comme le signalent Marriner et Morhange (2007), les taux de sédimentation dans les bassins portuaires sont systématiquement élevés car ils agissent comme des pièges à sédiments.

Les dragages mettent en exergue, bien qu'indirectement, des changements environnementaux susceptibles de participer au confinement du milieu portuaire (Goiran et al., 2014; Morhange et al., 2015). Au cours du XIII ${ }^{e}$ et XIV siècle, les prémices du petit âge de glace se font ressentir dans le Bas Languedoc. Les apports détritiques du Vistre, du Vidourle et du Rhône de La Ville sont plus importants, les cours d'eau sont sujets à une période d'activités hydro-sédimentaires associées à une fréquence et une amplitude accrue des crues (Berger et al., 2010), bien qu'encore modeste (Pichard et Roucaute, 2014). L'accélération des processus de sédimentation aux embouchures favorise l'alimentation des courants côtiers qui orientés vers le nord-ouest et chargés en sédiments, engraissent et allongent les cordons littoraux, et participent au remblaiement des graus. Poussée par les fleuves, l'avancée du trait de côte a donc été rapide entre le XIII ${ }^{e}$ et XIV ${ }^{e}$ siècle (Rey et al., 2009). Incomplète, cette image des dynamiques fluviales et marines contribue à mieux apprécier les différentes causes de l'envasement portuaire.

L'enclavement du port l'éloigne des grandes dynamiques laguno-marines. Le colmatage du bassin portuaire s'accompagne de la rétraction du plan d'eau. Comme Portus à Ostie (Goiran et al., 2010), ces éléments aboutissent nécessairement à une dégradation des qualités portuaires que ce soit en termes d'accessibilité, de modes de circulation sur des espaces de haut fonds et d'espaces de mouillages des navires.

6. Archives Nationales, J 896, n 22 : il s'agit d'une convention passée avec le génois Nicolas Cominelli, adressé par le sénéchal de Beaucaire Adam de Montcelard au roi Philippe le Bel, après le 16 octobre 1289 et dans lequel sont décrits les travaux d'aménagement du port d'AiguesMortes et du Môle.
Le colmatage du port ne signe pas nécessairement le déclin du port de la cité car même si l'accueil des navires à grand tirant d'eau devient impossible, le cabotage se pratique aisément et depuis longtemps dans ces environnements lagunaires.

Avant même le départ pour la septième croisade, le port fondé par Louis IX s'envasait. Pour faire face à ces problèmes, Louis IX aurait fait creuser le Canal Vieil (dont les carottes sédimentaires sont en cours d'analyses), afin de le relier à un plan d'eau plus profond (l'actuel étang du Ponant?) faisant office d'avant-port. Un avant port nécessaire pour accueillir les nefs royales au départ pour les croisades. Ce canal auraitil constitué la solution technique pour faire face à l'hypersédimentation du port d'Aigues-Mortes?

\section{Aigues-Mortes : la question des abords}

Dans les conditions architecturales liées à l'existence d'un dispositif de fondation sur radier et pieux, l'hypothèse de fossés défensifs traditionnels semble exclue. Nous entendons par ce terme, une excavation profonde aménagée au pied des fortifications, comme le cas se présente en bien des exemples castraux ou urbains situés en terrain favorable. La nécessité toutefois de conserver la superstructure basse implantée dans un contexte homogène exige que le terrain soit compact aux abords. Ceci selon le principe mécanique des pieux exerçant simultanément une pression verticale et latérale, fonctionnement au sein duquel tout vide exerce une action destructrice. L'idée de proximité de fossés creux est donc à éliminer.

À Aigues-Mortes, la version exploite la nature même du terrain et ce sont les marécages qui peuvent avoir naturellement constitué des défenses efficaces. Empêchant tout déplacement en raison de la consistance boueuse du fond et de la présence d'une végétation dense (nombreux débris contenus dans la tourbe prélevée), ces étendues entourant la ville nécessitaient d'être toutefois praticables pour les besoins de fonctionnement de la cité. Il est permis de penser, en l'absence de vestiges maçonnés, que ce sont des aménagements légers en bois implantés dans les contextes humides qui remplaçaient cette fonction. Amovibles par définition, les dispositifs pouvaient le cas échéant être condamnés momentanément, voire détruits, puis remplacés après une période d'assaut militaire. Il faut ainsi envisager la présence devant les grandes portes de pontons reliés à une digue ou talus périphérique surélevé, obtenu après un remblaiement massif. La question des contraintes naturelles est donc complexe car, dans le cas de la cité d'Aigues-Mortes, ces contraintes représentent aussi une opportunité de protection aux assauts, un obstacle naturel. La mobilité littorale, les plans d'eau peu profonds et les zones humides constituent des éléments de 
protection de la cité. Et en même temps, ces conditions environnementales ont favorisé le colmatage du port.

Un principe similaire peut s'appliquer au secteur méridional de la ville où la proximité de l'étang a nécessité la construction en dur de digues et de passerelles. La digue de la Peyrade témoigne du premier cas de figure. Aux abords immédiats de la ville, un environnement lagunaire et palustre doit être envisagé. Tout aussi impraticables que les zones de défenses assimilées à des fossés, les berges ont nécessité la construction de dispositifs en bois longeant les courtines. Ancrées dans le sol, les constructions n'exigent pas d'être nécessairement ancrées dans les maçonneries ce qui explique l'absence dans les parements de trou de scellement. Cette absence vérifiée dans l'emprise réduite des sondages pourrait toutefois être confirmée ailleurs. Notons que la découverte hors stratigraphie, et dans les contextes noyés inaccessibles, de pièces de bois peut illustrer cette thématique. La restitution présumée de passerelles permet de faire le lien dans les élévations avec les galeries de circulation hautes et périphériques placées au sommet des élévations.

La présence de structure de bois surélevée par rapport au niveau de la nappe et qui surmontait le niveau du plan d'eau pourrait expliquer la situation en hauteur des anneaux d'amarrage (à 2,60 m au-dessus du radier de fondation) qui prennent toute leur valeur évocatrice avec la restitution de ces aménagements de type portuaire. La valeur historique de ces témoignages, parfois mise en doute, ne peut être écartée en raison de leur nombre (18 emplacements identifiés) et de la suggestion apportée par les noms donnés aux portes situées sur cette face tournée vers la mer (Porte des Galions, Porte de la Marine).

L'étude de la cité d'Aigues-Mortes se poursuit pour reconstituer de façon plus fine et fiable la paléogéographie médiévale du port d'Aigues-Mortes (campagne électro magnétique et résistivité électrique). La multiplication des proxys (analyse des marqueurs sédimentologique, palynologique et ostracologique) apportera des éléments essentiels (salinité, profondeur, espèces végétales...) dans la caractérisation des dynamiques environnementales et paysagères au Moyen-Âge. Enfin, les études sur les principes de conception des fondations des remparts, les échanges commerciaux avec l'Espagne catalane, la question du bois...sont autant de nouvelles perspectives de recherches sur ce site mal connu.

\section{Remerciements}

Le programme Collectif de Recherche « Géoarchéologie des ports médiévaux d'Aigues-Mortes " est soutenu par la Direction Régionale des Affaires Culturelles LanguedocRoussillon, le Service Régional de l'Archéologie, l'université Paul-Valéry-Montpellier 3, les Monuments Nationaux et la Mairie d'Aigues-Mortes. Il réunit des collègues de différents horizons que nous tenons à remercier pour leur contribution : Jean-Philippe Cherel, Nicolas Faucherre, Lucie Galano, Marie-Pierre Jézégou, Claude Raynaud, Bernard Aubert. Nous remercions également l'Institut de Recherche et de Développement (IRD) ainsi que l'association "Sur Les Pas de Saint Louis " pour leur soutien logistique. Enfin, nous remercions Lucie Chabal et Armelle Gardeisen pour leur aide précieuse dans l'identification des bois et autres restes faunistiques et Jean-Philippe Degay et Gaël Piques (UMR 5140) pour le soutien logistique lors des journées de carottages. Les auteurs remercient les deux relecteurs pour leurs commentaires et suggestions.

\section{Bibliographie}

Anthony E.J., Marriner N., Morhange C., 2014. Human influence and the changing geomorphology of Mediterranean deltas and coasts over the last 6000 years: From progradation to destruction phase?, Earth-Science Reviews, 139, p. 336-361.

Arnaud-Fassetta G., Carcaud N., Castanet C., Salvador P. G., 2010. Fluviatile palaeoenvironments in archaeological context: Geographical position, methodological approach and global change - Hydrological risk issues. Quaternary International, 216, p. 93-117.

Bagan G., Gailledrat E., Jorda C., 2010. Approche historique de la géographie des comptoirs littoraux à l'Âge du Fer en Méditerranée occidentale à travers l'exemple du port de Lattara (Lattes, Hérault). Quaternaire, 21 (1), p. 85-100.

Bellet M.-E., Florençon P., 2001. La cité d'Aigues-Mortes, Éditions du Patrimoine, coll. "Itinéraires ", 2001.

Berger J.-F., Blanchemanche P., Reynes C., Sabatier P., 2010. Dynamiques fluviales en basse vallée du Vidourle au cours des six derniers siècles. Quaternaire, vol. 21/1, p. 27-41.

Bony G., Morhange C., Kaniewski D., Marriner N., 2014. Contraintes et potentialités naturelles des bassins portuaires antiques, essai de typologie. In L. Mercuri, R. González Villaescusa et F. Bertoncello (éd.), $34^{\mathrm{e}}$ rencontres internationales d'archéologie et d'histoire d'Antibes. Antibes, Éditions APDCA, p. 95-107. 
Bronk R C., Dee M., Lee S., Nakagawa T., Staff R., 2010. Developments in the calibration and modelling of radiocarbon dates. Radiocarbon, 52(3), p. 953-961.

Chabal L., 1997. Forêts et sociétés en Languedoc (Néolithique final, Antiquité tardive) : L'anthracologie, méthode et paléoécologie. Paris.

Chabal L. avec la collaboration de Fiches J.-L., Mathieu V., Falquet A.-V., 2012. Poutres, appareillages et mobiliers en bois. $R A N$, supplément 42, p. 65-127.

Florençon P., 1996. Aigues-Mortes et la Méditerranée au Moyen Âge, Recherches sur le port et choix de documents. Mémoire pour la Caisse Nationale des Monuments Historiques et des Sites.

Goiran J.-P., Tronchère H., Salomon F., Carbonel P., Djerbi H., Ognard, C., 2010. Palaeoenvironmental reconstruction of the ancient harbors of Rome: Claudius and Trajan's marine harbors on the Tiber delta. Quaternary International, 216, p. 3-13.

Goiran J.-P., Salomon F., Mazzini I., Bravard J.-P., Pleuger E., Vittori C., Boetto G., Christiansen J., Arnaud P., Pellegrino A., Pepe C., Sadori L., 2014. Geoarchaeology confirms location of the ancient harbour basin of Ostia (Italy). Journal of Archaeological Science, 41, p. 389-398.

L'Homer A., 1987. Notice explicative, Carte géologique. France (1/50000), feuille Arles (992). BRGM, Orléans.

L'Homer A., 1993. Notice explicative, Carte géologique. France (1/50000), feuille Grau du Roi (1017), BRGM, Orléans.

Mazauric F., 1910. Mémoires de l'Académie de Nîmes. $7^{\mathrm{e}}$ série, 33.

Markiewicz C., 2011. Aigues-Mortes (30). Étude et sondages archéologiques, Porte de la Reine et Porte de la Marine. Rapport/ DFS, DRAC Languedoc-Roussillon, Conservation régionale des Monuments historiques et Service régional de l'archéologie, Montpellier.

Marriner N., Morhange C., 2006. Geoarchaeological evidence for dredging in Tyre's ancient harbour, Levant. Quaternary Research, 65, p. 164-171.

Marriner N., Morhange C., 2007. Geoscience of ancient Mediterranean harbours. Earth Science Reviews, n 3, Vol. 80, p. 137-194.

Marriner N., Morhange C., 2010. Mind the (stratigraphic) gap: Roman dredging in ancient Mediterranean harbours. Bollettino di Archeologia on Line, volume B7, p. 23-32.

Ministère des Affaires culturelles, 1973. Inventaire général des monuments et des richesses artistiques de la France, Commission régionale du Languedoc - Roussillon. Gard. Canton d'AiguesMortes, Paris, Imprimerie nationale.

Morhange C., Laborel J., Hesnard A., 2001. Changes of relative sea level during the past 5000 years in the ancient harbour of Marseilles, Southern France, Palaeogeography, Palaeoclimatology, Palaeooecology, 166, p. 319-329.
Morhange C., Marriner N., 2009. Roman dredging in ancient Mediterranean harbours, Bollettino di Archeologia On Line, vol. spe. IAAC.

Morhange C., Marriner N., 2010. Palaeo-hazards in the coastal Mediterranean: a geoarchaeological approach. In I.P. Martini, W. Chesworth (eds.), Landscapes and Societies. Springer, Dordrecht, The Netherlands, p. 223-234.

Morhange C., Marriner N., Baralis A., Blot M.L., Bony G., Carayon N., Carmona P., Flaux C., Giaime M., Goiran J.-P., Kouka M., Lena A., Oueslati A., Pasquinucci M., Porotov A., 2015. Dynamiques géomorphologiques et typologie géoarchéologique des ports antiques en contextes lagunaires. Quaternaire, 26, (2), p. 117-139.

Pagézy J. 1879. Mémoires sur le port d'Aigues-Mortes (AiguesMortes). Librairie Hachette et Cie, 1879-1886, Paris.

Pichard G., Roucaute E., 2014. Pluies et crues en bas Rhône et caractérisation du Petit Age de Glace. Méditerranée, 122, p. 31-42.

Provansal M., Morhange C., Vella C., 1995. Impact des anthropiques et contraintes naturelles sur les sites portuaires antiques de Marseille et de Fos. Acquis méthodologiques. Méditerranée, 3-4, p. 93-100.

Provost M., 1999. Carte archéologique de la Gaule 30/2 - Le Gard. Académie des Inscriptions et Belles-Lettres.

Py M., Roure R., 2002. Le Cailar (Gard) : Un nouveau comptoir lagunaire protohistorique au confluent du Rhôny et du Vistre. Documents d'archéologie méridionale, 25, p. 171-214.

Raynaud C. (dir.), 1999. La vallée du Vidourle, géoarchéologie d'un paysage méditerranéen. Programme Collectif Régional, UMR 154 de Montpellier/Lattes, inédit.

Reimer P.J. et al., 2009. IntCal09 and Marine09 radiocarbon age calibration curves, 0-50,000 years cal BP. Radiocarbon 51(4), p. 1111-1150.

Rey T., 2006. Dynamiques hydro-sédimentaires en Petite Camargue à l'Holocène. Thèse de l'Université Montpellier 3 - spécialité : Géographie Physique.

Rey T., Lefevre D., Vella C., 2009. Deltaic plain development and environmental changes in the Rhône Delta (France) in the past 2000 years. Quaternary Research, 71, p. 284-294.

Rey T., 2010. Modalités du remblaiement sédimentaire des paléochenaux holocènes identifiés dans la partie occidentale du delta du Rhône (Petite Camargue, France). Quaternaire, 21, (1), p. 101-111.

Rey T., Roure R., Campan E., 2015. Les « lacs » des Celtes : synthèse de l'évolution paléogéographique et environnementale des littoraux du Languedoc oriental depuis la protohistoire. In Actes du $37^{\mathrm{e}}$ Colloque International de l'AFEAF, Montpellier, vol. 1, p. 21-34.

Sanchez C., Faïsse C., Jézégou M.-P., Mathé V., 2014. Le système portuaire de Narbonne antique : approche géoar- 
chéologique. In Mercuri L., González Villaescusa R., Bertoncello F. (eds.), $34^{\mathrm{e}}$ rencontres internationales d'archéologie et d'histoire d'Antibes. Éditions APDCA, Antibes, p. $125-136$.

Tuente U., Piepenburg D., Spindler M., 2002. Occurrence and settlement of the common ship worm Teredo navalis (Bivalvia : Teredinidae). In Bremer haven harbours, northern Germany, Helgoland. Marine Research, 56, p. 87-94.
VAYsSETTES J.-L., 1996. Les entrées de céramiques d'après le livre des comptes du port d'Aigues-Mortes. In Poteries d'Oc, céramiques languedociennes VII'-XVII siècles, Narration Éditions, 1995, p. 113-115.

Vella C., Fleury T.J., Raccasi G., Provansal M., Sabatier F., Bourcier M., 2005. Evolution of the Rhône delta plain in the Holocene. Marine Geology, p. 222-223, p. 235-265. 
Figure 8 : Thomas Delbey et al., Amphores vinaires Gauloise 4 de Narbonnaise (France) exportées à Carthagène (Espagne) ? Analyse statistique exploratoire multidimensionnelle de données géochimiques (p. 196)

Figure 1 : Tony Rey et al., La cité médiévale d'AiguesMortes (Gard) : nouvelles données géomorphologiques et archéologiques sur un site méconnu (p. 204)

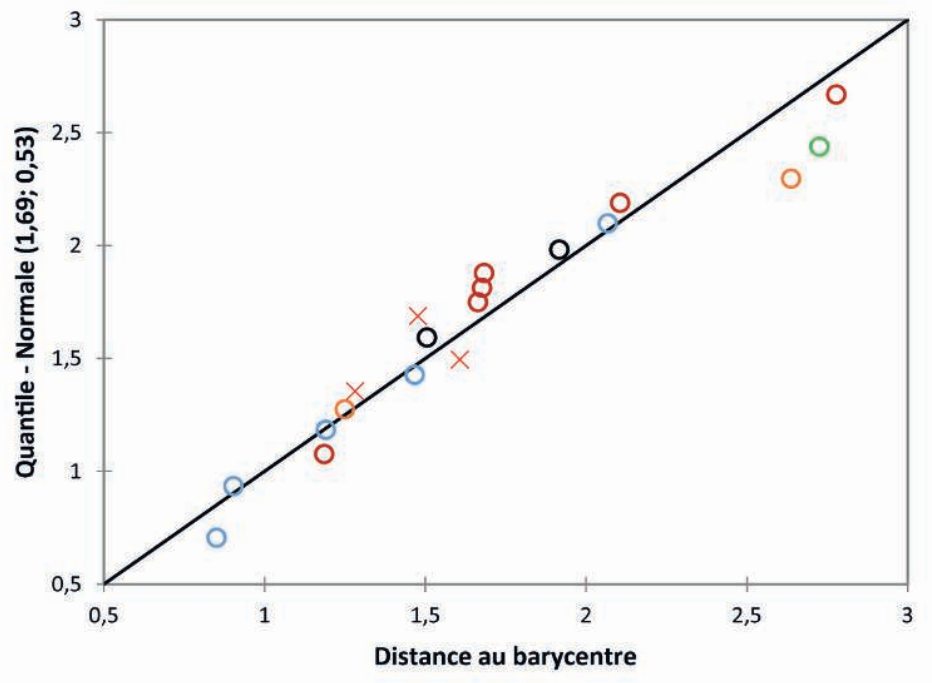

$\begin{array}{ll}\text { OMeynes OMontfrin } & \text { Saint-Gilles } \\ \text { OBeaucaire OOraison } \times \text { Carthagène }\end{array}$

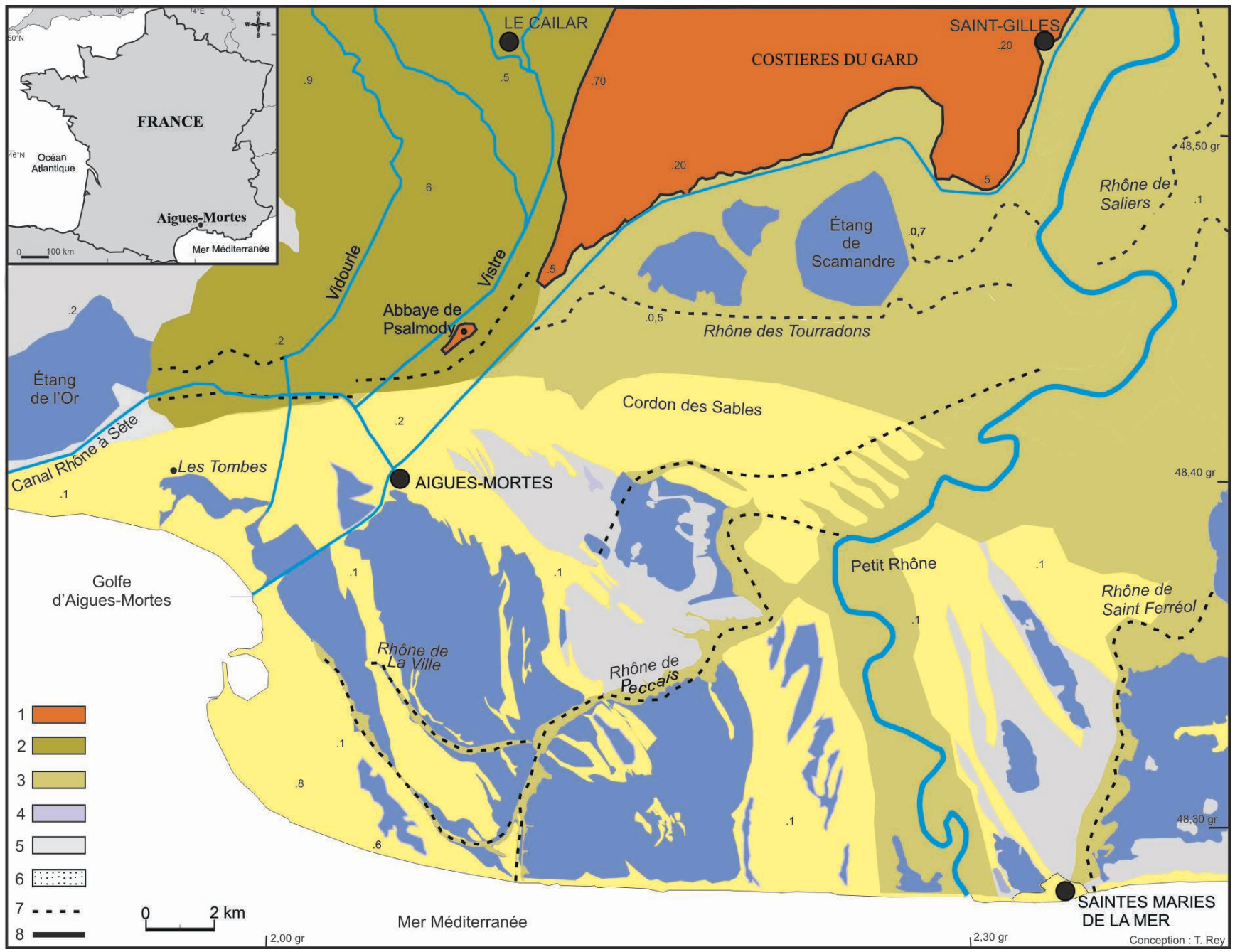




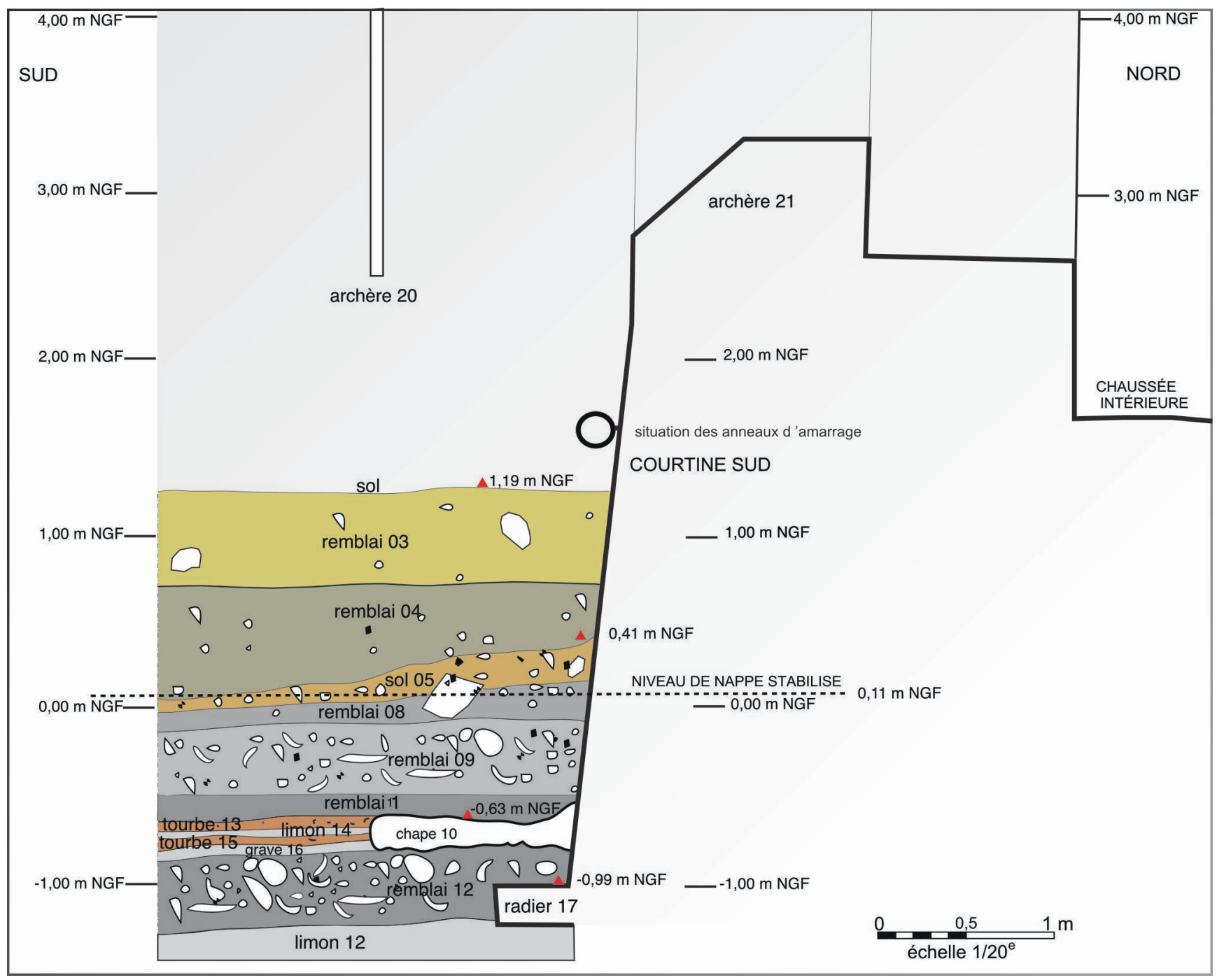

Figure 3 : Tony Rey et al., La cité médiévale d'Aigues-Mortes (Gard) : nouvelles données géomorphologiques et archéologiques sur un site méconnu (p. 206)

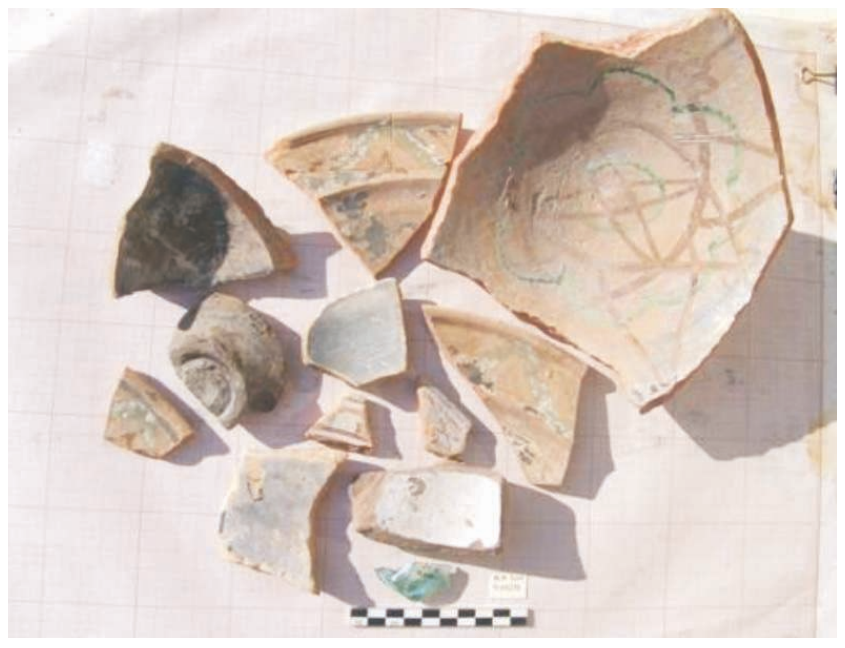

Figure 5 : Tony Rey et al., La cité médiévale d'Aigues-Mortes (Gard) : nouvelles données géomorphologiques et archéologiques sur un site méconnu (p. 207)

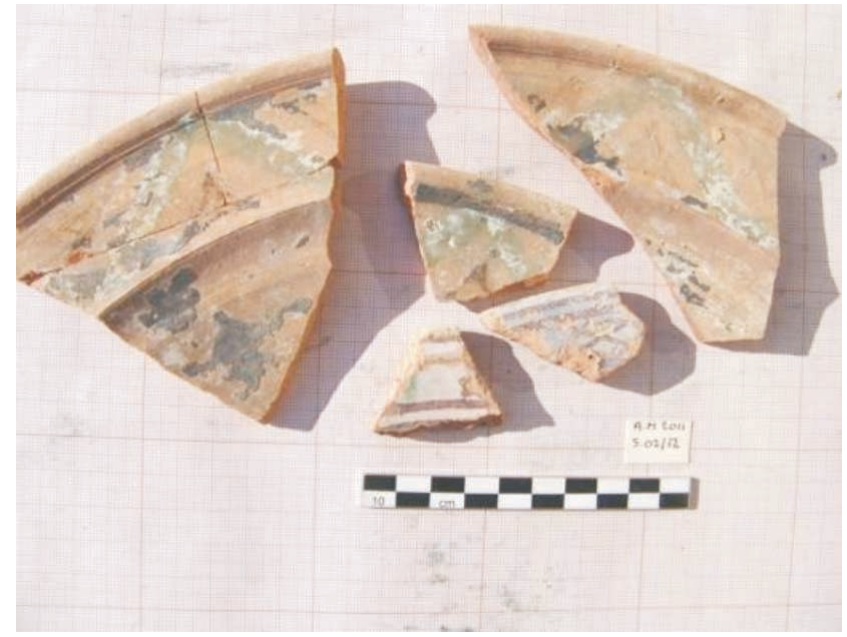

Figure 6 : Tony Rey et al., La cité médiévale d'Aigues-Mortes (Gard) : nouvelles données géomorphologiques et archéologiques sur un site méconnu (p. 207) 


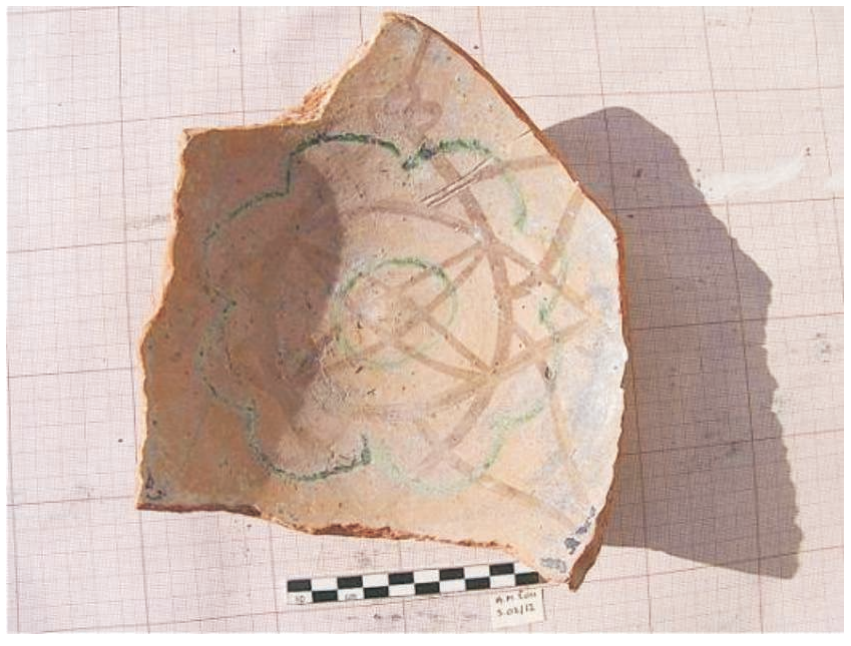

Figure 7 : Tony Rey et al., La cité médiévale d'Aigues-Mortes (Gard) : nouvelles données géomorphologiques et archéologiques sur un site méconnu (p. 208)

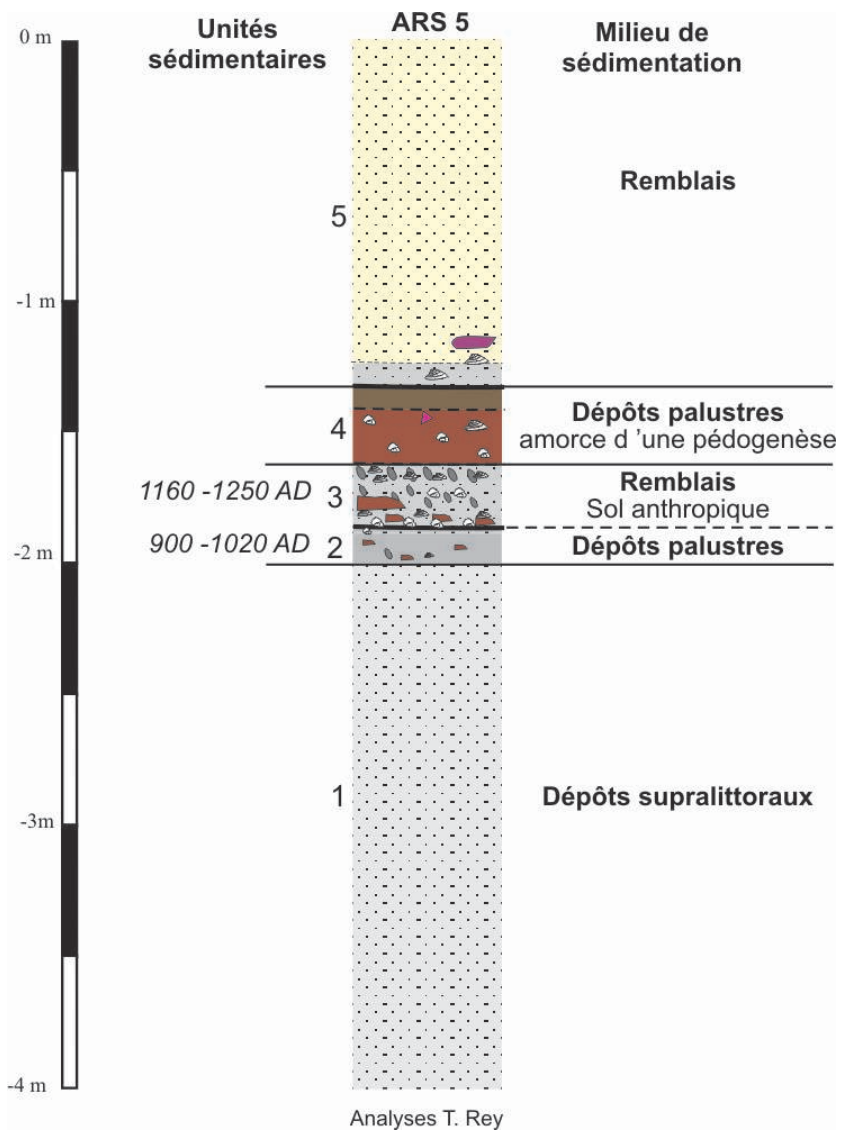

Figure 10 : Tony Rey et al., La cité médiévale d'Aigues-Mortes (Gard) : nouvelles données géomorphologiques et archéologiques sur un site méconnu (p. 210)

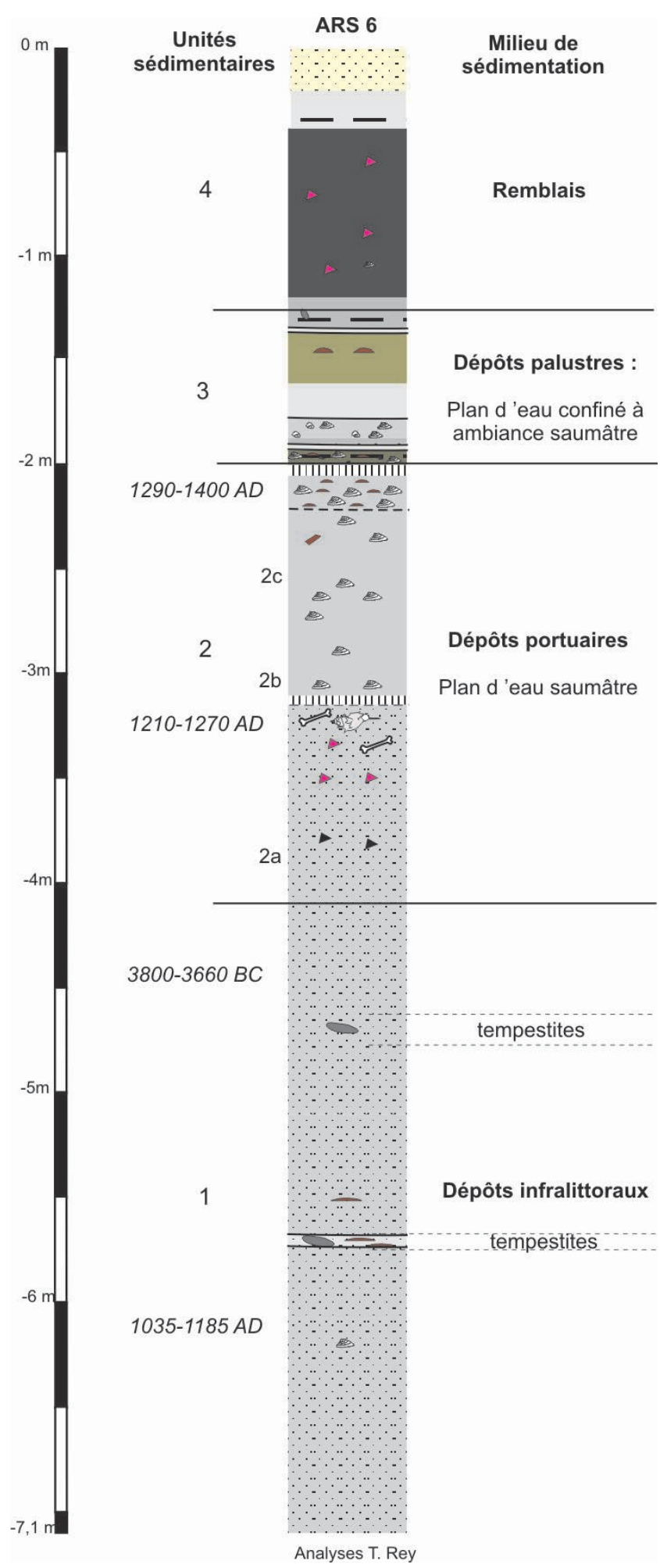

Figure 11 : Tony Rey et al., La cité médiévale d'Aigues-Mortes (Gard) : nouvelles données géomorphologiques et archéologiques sur un site méconnu (p. 211) 